Published in final edited form as:

Nat Immunol. 2017 March ; 18(3): 293-302. doi:10.1038/ni.3655.

\title{
Chronic signaling via the metabolic checkpoint kinase mTORC1 induces macrophage granuloma formation and marks sarcoidosis progression
}

\author{
Monika Linke ${ }^{1}$, Ha Thi Thanh Pham ${ }^{1}$, Karl Katholnig ${ }^{1}$, Thomas Schnöller ${ }^{1}$, Anne Miller ${ }^{2}$, \\ Florian Demel $^{1}$, Birgit Schütz ${ }^{1}$, Margit Rosner ${ }^{1}$, Boris Kovacic ${ }^{1}$, Nyamdelger Sukhbaatar ${ }^{1}$, \\ Birgit Niederreiter ${ }^{3}$, Stephan Blüml ${ }^{3}$, Peter Kuess ${ }^{4}$, Veronika Sex ${ }^{5}$, Mathias Müller ${ }^{6}$, Mario \\ Mikula $^{1}$, Wolfram Weckwerth ${ }^{7}$, Arvand Haschemi ${ }^{2}$, Martin Susani ${ }^{8}$, Markus \\ Hengstschläger ${ }^{1}$, Michael J Gambello ${ }^{9}$, and Thomas Weichhart ${ }^{1,{ }^{*}}$ \\ ${ }^{1}$ Center of Pathobiochemistry and Genetics, Institute of Medical Genetics, Medical University of \\ Vienna, 1090 Vienna, Austria \\ ${ }^{2}$ Department of Laboratory Medicine (KILM), Medical University of Vienna, 1090 Vienna, Austria \\ ${ }^{3}$ Division of Rheumatology, Department of Internal Medicine III, Medical University of Vienna, \\ 1090 Vienna, Austria \\ ${ }^{4}$ Department of Radiation Oncology, Division of Medical Radiation Physics, Medical University of \\ Vienna, 1090 Vienna, Austria \\ ${ }^{5}$ Institute of Pharmacology and Toxicology, Department for Biomedical Sciences, University of \\ Veterinary Medicine Vienna, 1210 Vienna, Austria \\ ${ }^{6}$ Biomodels Austria and Institute of Animal Breeding and Genetics, University of Veterinary \\ Medicine, 1210 Vienna, Austria \\ ${ }^{7}$ Department of Ecogenomics and Systems Biology, Vienna Metabolomics Center (VIME), \\ University of Vienna, 1090 Vienna, Austria \\ ${ }^{8}$ Clinical Institute of Pathology, Medical University of Vienna, 1090 Vienna, Austria \\ ${ }^{9}$ Department of Human Genetics, Emory University, Atlanta, Georgia 30322, United States
}

\author{
Abstract \\ Users may view, print, copy, and download text and data-mine the content in such documents, for the purposes of academic research, \\ subject always to the full Conditions of use:http://www.nature.com/authors/editorial_policies/license.html\#terms \\ *Correspondence: thomas.weichhart@meduniwien.ac.at. \\ Accession codes. Gene Expression Omnibus Microarray data have been deposited under accession code GSE77075. \\ Data Availability Statement \\ Further information regarding data and reagents used is available by request to the corresponding author. \\ Author Contributions \\ Conceptualization, M.L., and T.W.; Investigation: M.L., H.T.T.P., K.K., T.S., A.M., F.D., B.S., M.R., B.K., N.S., B.N., P.K., and T.W.; \\ Resources: S.B., V.S., M.Mi., M.Mü., W.W., A.H., M.S., M.H., M.J.G. and T.W.; Writing - Original Draft: T.W.; Writing - Review \& \\ Editing: all authors; Supervision, T.W. \\ Competing Financial Interests \\ The authors declare no competing financial interests.
}


Aggregation of hypertrophic macrophages constitutes the basis of all granulomatous diseases such as tuberculosis or sarcoidosis and is decisive for disease pathogenesis. However, macrophageintrinsic pathways driving granuloma initiation and maintenance remain elusive. Here we show that activation of the metabolic checkpoint kinase mTORC1 in macrophages by deletion of $T s c 2$ was sufficient to induce hypertrophy and proliferation resulting in excessive granuloma formation in vivo. TSC2-deficient macrophages formed mTORC1-dependent granulomatous structures in vitro and showed constitutive proliferation mediated by the neo-expression of cyclin-dependent kinase 4 (CDK4). Moreover, mTORC1 promoted metabolic reprogramming via CDK4 towards increased glycolysis, while simultaneously inhibiting NF- $\mathrm{kB}$ signaling and apoptosis. Inhibition of mTORC1 induced apoptosis and completely resolved granulomas in myeloid TSC2-deficient mice. In human sarcoidosis patients mTORC1 activation, macrophage proliferation, and glycolysis were identified as hallmarks that correlated with clinical disease progression. Collectively, TSC2 maintains macrophage quiescence and prevents mTORC1-dependent granulomatous disease with clinical implications for sarcoidosis.

Granulomas are compact aggregates of mature macrophages with an increased cytoplasmic size whose membranes become interlaced leading them to be called epithelioid cells1. They are usually formed and maintained in response to the continuous presence of either infectious stimuli such as bacteria, fungi, protozoa, trematodes and viruses, or in response to non-infectious foreign-body particles. Tuberculosis or schistosomiasis are prime examples of an infectious granulomatous disease, whereas non-infectious granuloma formation is observed in sarcoidosis, Crohn's disease, primary biliary cirrhosis but also in neoplasias2-6. Sarcoidosis is an enigmatic granulomatous disease of unknown etiology that most commonly affects the lung, lymph nodes, skin, and liver5. The onset is gradual from an asymptomatic state to a progressive disease that persists in about one-third of patients and can become life threatening5,7. Molecular signals or pathways that control disease progression are largely undefined8,9. When treatment is required, corticosteroids are usually recommended, but they are associated with significant side effects7. Due to the unknown etiology, there is currently no therapeutic approach targeting the pathogenetic mechanisms.

Because of the major clinical role of granulomas in disease pathologies, the morphological properties of granuloma formation have been extensively studied. Generally, the principal and initiating element of a granuloma is the macrophage, for example alveolar macrophages induce granuloma formation after mycobacterial infection in the lung2,10,11. However, many other cell types are also found later on in mature granulomas such as neutrophils, dendritic cells, B and T cells, NK cells, and fibroblasts6,11,12. These cells mostly surround the basic macrophage core to form a highly organized granulomatous structure. However, immunocompromised SCID mice are still able to form granulomas after mycobacteria infection indicating the non-essential role of adaptive immunity in granuloma initiation 10 . Hence, the adaptive immune system contributes more to granuloma organization and protection to limit dissemination of the infection and injury for the host2,13,14. Similarly, although factors such as TNF, IFN- $\gamma$, IL-12, IL-10, CCL2, HMOX1, or MMP9 regulate granuloma organization and pathogen susceptibility in infectious granulomatous diseases, initial granuloma formation occurs independently of these signals 10,15,16. Hence, the fundamental molecular pathways underlying macrophage transformation to hypertrophic 
epithelioid cells that aggregate, initiate, and maintain macrophage granulomas are still unknown.

The mechanistic target of rapamycin (mTOR) signaling pathway senses and integrates a range of environmental signals to regulate cellular metabolism and cell growth in many cell types and is implicated in an increasing number of pathological conditions17. Tuberous sclerosis 2 (TSC2) is a tumor suppressor that forms a heterodimeric complex with TSC1 and negatively regulates mTOR complex 1 (mTORC1)18. The mTOR pathway, as an energy and nutritional sensor, is exquisitely suited to regulate processes such as glycolysis or mitochondrial metabolism to influence effector responses 19,20. Activation of mTORC1 regulates inflammatory immune responses in innate immune cells such as monocytes, macrophages, and dendritic cells21. Genetic manipulation of the mTOR pathway in mice alters macrophage polarization and the production of inflammatory and immunomodulatory cytokines22-24. However, potential additional functions for the mTORC1 pathway in macrophages are unknown. Here, we found that activation of mTORC1 by deletion of $T s c 2$ in macrophages is sufficient to initiate and maintain granulomas. Molecularly we find that mTORC1 inhibits apoptosis and induces macrophage proliferation by inducing the expression of the cell cycle kinase CDK4 and metabolic reprogramming. These mechanistic features are also associated with disease progression in human sarcoidosis.

\section{Results}

\section{Myeloid TSC2-deficiency promotes granuloma formation}

We aimed to define the role of mTORC1 in macrophages by generating a mouse model for constitutive mTORC1 activation in myeloid cells by deletion of its upstream inhibitor TSC217. Therefore, we crossed $T s c 2^{\mathrm{fl} / \mathrm{fl}}$ mice 25 with transgenic mice expressing Cre under the control of the lysozyme promoter (designated $T s c 2^{\mathrm{fl} / \mathrm{fl}} L y z 2$-Cre). Conditional myeloid deletion of $T s c 2$ induced the formation of non-caseating (non-necrotizing) granulomatous aggregates, predominantly found in the lung and liver but also in lymph nodes of 3 monthold $T s c 2^{\mathrm{fl} / \mathrm{fl}} L y z 2$-Cre mice that were absent in control mice ( $\left.T s c 2^{\mathrm{fl} / \mathrm{fl}}\right)$ (Fig. 1a and Supplementary Fig. 1a-c). The hypertrophic and epithelioid cell clusters stained positive for the macrophage marker Mac-2 and showed strong phosphorylation of S6 (p-S6), a hallmark of mTORC1 activation (Fig. 1b). Although the hypertrophic cells expressed some myeloperoxidase, they could be clearly distinguished from neutrophils (Supplementary Fig. 1d). The phenotype worsened by 6 months and was accompanied by strong inflammatory infiltrates (Fig. 1c). At about 4 months $T s c 2^{21 / 1 / 1} L y z 2$-Cre mice developed swollen paws and tails resulting from excessive granulomas that were largely composed of hypertrophic Mac-2 and F4/80-positive macrophages (Fig. 1d,e). We also found profound hypertrophy in $T s c 2^{21 / / 1} L y z 2$-Cre peritoneal macrophages (Fig. 1f). Transplantation of $T s c 2^{\mathrm{fl} / \mathrm{fl}} L y z 2$-Cre but not $T s c 2^{\mathrm{t} / \mathrm{fl}}$ bone marrow recapitulated granuloma formation in the lung and liver suggesting that hypertrophic macrophage accumulation represents a cell-autonomous phenotype (Fig. $1 \mathrm{~g})$.

Alveolar macrophages have a central role in human granulomatous lung lesions8,26. We observed a population of hypertrophic lung cells in $T s c 2^{\mathrm{fl} / \mathrm{fl}} L y z 2$-Cre but not $T s c 2^{\mathrm{fl} / \mathrm{fl}}$ mice (Fig. 1h and Supplementary Fig. 2a). Phenotypic analysis using a previously established 
protocol27 showed that these cells comprise hypertrophic alveolar macrophages but not typical interstitial macrophages, infiltrating Ly6 $\mathrm{C}^{\text {hi }}$ monocytes, or other known myeloid or lymphoid immune cells (Supplementary Fig. 2b,c). Functional characterization of the inflamed lung showed enhanced mRNA expression of Lgals3 (encoding Mac-2), Chil3, Igf1, IIIO, and Mrc1 but reduced expression of Nos2 (encoding iNOS) indicating that the macrophages have an enhanced M2-like polarization (Fig. 1i). The polarization profile, however, was not strongly maintained in bone marrow-derived macrophages (BMDM) in vitro (Supplementary Fig. 2d). Therefore, hypertrophic M2-like macrophages progressively accumulate in various tissues of $T s c 2^{\text {fl/fl }} L y z 2$-Cre mice in a cell-autonomous manner and form granulomas that disturb normal tissue homeostasis.

\section{TSC2 prevents cluster formation and proliferation}

To identify how deletion of TSC2 promotes hypertrophic granuloma formation, we generated BMDM from $T s c 2^{2^{1} / / f 1}$ and $T s c 2^{\mathrm{fl} / f 1} L y z 2$-Cre mice. As expected $T s c 2^{\mathrm{fl} / \mathrm{fl}} L y z 2$-Cre BMDM showed constitutive activation of the mTORC1 pathway indicated by phosphorylation of the downstream effector S6 and activation of 4E-BP1 that could both be blocked by rapamycin (Fig. 2a and Supplementary Fig. 3a). We also found that the activity of mTORC2, indicated by phosphorylation of Akt serine 473 - p-Akt (S473) - was decreased in these cells (Fig. 2a). In addition, the macrophage growth factor CSF1 and lipopolysaccharide (LPS) induced phosphorylation of TSC2 on Ser939 accompanied by mTORC1 activation in wild-type BMDM (Supplementary Fig. 3b). Expression of the myeloid-specific surface markers F4/80, CD11b, CD115, Gr1, or CD11c was similar between $T s c 2^{\mathrm{fl} / \mathrm{fl}}$ and $T s c 2^{\mathrm{fl} / \mathrm{fl}} L y z 2$-Cre macrophages suggesting that loss of TSC2 does not impair macrophage differentiation (Fig. 2b). However, $T s c 2^{\text {fl/fl }} L y z 2$-Cre BMDM formed granulomatous cell clusters after prolonged culture in vitro and had increased granularity and cell size (Fig. 2c,d and Supplementary Fig. 3c,d) reminiscent of the hypertrophic cells observed in vivo. Notably, rapamycin treatment reversed clustering as well as the hypertrophic phenotype of BMDM and of isolated lung macrophages from $T s c 2^{\mathrm{fl} / \mathrm{fl}} L y z 2$-Cre mice (Fig. 2c and Supplementary Fig. 3c,e,f). $T s c 2^{\mathrm{fl} / \mathrm{fl}} L y z 2$-Cre dendritic cells or human and mouse fibroblasts, where $T s c 2$ was knocked down, did not show granulomatous cluster formation indicating specificity towards the macrophage lineage (Supplementary Fig. 3g,h). We noticed higher numbers of $T s c 2^{2 \mathrm{l} / / \mathrm{f}} L y z 2$-Cre BMDM compared to $T s c 2^{\mathrm{fl} / f \mathrm{l}}$ cells on day 7 of culture suggestive of enhanced proliferation (Supplementary Fig. 4a). Cell cycle analysis verified that CSF1-deprived $T s c 2^{\mathrm{fl} / \mathrm{fl}} L y z 2$-Cre macrophages had enhanced cell cycling indicated by higher percentages of cells in the $\mathrm{S}$ and G2/M phase. (Fig. 2e and Supplementary Fig. 4b). This proliferative phenotype was also observed in $T_{s c 2^{\mathrm{fl}} / \mathrm{fl}} L y z 2$-Cre peritoneal macrophages in vivo (Fig. 2f). Additionally, there were high numbers of Ki67positive macrophages in the granulomatous lung and skin lesions of $T_{s c} 2^{\mathbb{f l} / f 1} L y z 2$-Cre mice indicating that macrophage proliferation contributes to the TSC2-dependent granuloma formation (Fig. $2 \mathrm{~g}$ and Supplementary Fig. 4c). Finally, the macrophage growth factor CSF1 induced mTORC1-dependent proliferation much stronger in $T s c 2^{21 / 1 / 1} L y z 2$-Cre macrophages compared to $T s c 2^{\mathrm{fl} / \mathrm{fl}}$ cells (Fig. $2 \mathrm{~h}$ and Supplementary Fig. 4d). These results show that deletion of $T_{s c 2}$ in macrophages is sufficient to spontaneously induce hypertrophy, granulomatous aggregation and hyperproliferation in vitro and in vivo. 


\section{TSC2 regulates macrophage quiescence versus inflammation}

To decipher the mechanisms that contributed to this phenotype, we performed transcriptome analysis of $T s c 2^{\text {fl/fl }}$ and $T s c 2^{\mathrm{fl} / f 1} L y z 2$-Cre macrophages deprived of CSF1. We identified 401 genes that were upregulated in $T s c 2^{\mathrm{fl} / \mathrm{fl}} L y z 2$-Cre macrophages more than 2-fold, whereas 426 were downregulated in these cells compared to $T s c 2^{\mathrm{fl} / \mathrm{fl}}$ macrophages (Fig. 3a). Gene set enrichment analysis (GSEA) showed that E2F transcription factor targets were the most highly enriched gene set in $T s c 2^{\mathrm{fl} / \mathrm{fl}} L y z 2$-Cre macrophages suggesting that the TSC2mTORC1 pathway is a major cell cycle regulator in macrophages (Fig. 3b,c). We also found E2F1 expressed slightly higher in $T s c 2^{\mathrm{fl} / \mathrm{fl}}$ Lyz2-Cre BMDM compared to their controls (Supplementary Fig. 4e). Moreover, a proliferative self-renewal gene signature28 was strongly enriched in $T s c 2^{\mathrm{fl} / \mathrm{fl}}$ Lyz2-Cre cells (Supplementary Fig. 4f). In contrast, the inflammatory NF- $\kappa \mathrm{B}$ response and apoptosis were augmented in $T s c 2^{\mathrm{fl} / \mathrm{fl}}$ cells (Fig. $3 \mathrm{~d}, \mathrm{e}$ ). Analysis of transcription factor binding sites corroborated that genes containing E2F binding sites were highly enriched in $T_{s c} 2^{\mathrm{fl} / \mathrm{fl}} L y z 2$-Cre macrophages, whereas NF- $\kappa \mathrm{B}$ binding sites were enriched in $T s c 2^{\mathrm{fl} / \mathrm{fl}}$ cells (Fig. 3f). Hence, active TSC2 limits E2F activation in macrophages but promotes NF- $\mathrm{kB}$ signaling and apoptosis to balance proliferation, selfrenewal and inflammatory properties.

\section{CSF1 induces CDK4 expression via TSC2/mTORC1 in macrophages}

Subsequently, we wanted to investigate the regulation of apoptosis and E2F activation in TSC2-deficient macrophages in more detail. Indeed, we found reduced apoptosis in CSF1deprived $T s c 2^{\text {fl/fl }} L y z 2$-Cre macrophages shown by a rapamycin-sensitive decrease of cleaved caspase 3 (Fig. 4a). Molecularly, this was associated with enhanced expression of the anti-apoptotic molecules Bcl-2 and survivin and with reduced protein expression of proapoptotic PDCD4 (Fig. 4a and Supplementary Fig. 4g). On the other hand, the protein expression of cyclins $\mathrm{D} 1$ and $\mathrm{A}$, the phosphorylation of $\mathrm{Rb}$, and the degradation of the negative cell cycle regulator $\mathrm{p} 27^{\mathrm{Kip} 1}$ in CSF1-starved $T s c 2^{\mathrm{fl} / \mathrm{fl}}$ Lyz2-Cre BMDM were indicative of active cell cycling (Supplementary Fig. 5a). This expression pattern was reversed by rapamycin and thus dependent on mTORC1 (Supplementary Fig. 5a). D type cyclins bind to CDK4, the kinase that drives G1 to $\mathrm{S}$ transition by activating E2F29. There is evidence that expression of CDK4 is constitutive and not modified by environmental stimuli30. However, we noted enhanced protein expression of CDK4 in $T s c 2^{21 / f 1} L y z 2-C r e$ macrophages that was sensitive to mTORC1 inhibition (Fig. 4b). Even more strikingly, CSF1 stimulated CDK4 expression in $T s c 2^{\mathrm{fl} / \mathrm{fl}}$ or C57BL/6J BMDM cells in a dosedependent manner (Fig. 4c, and Supplementary Fig. 5b-d). However, CSF1 only marginally influenced CDK4 in $T s c 2^{\mathrm{fl} / \mathrm{fl}} L y z 2$-Cre macrophages, where CDK4 expression was already high (Fig. 4c). CDK4 expression in the hypertrophic macrophages of the $T s c 2^{\mathrm{fl} / \mathrm{fl}} L y z 2$-Cre mice was also enhanced in vivo (Fig. 4d and Supplementary Fig. 5e). Furthermore, mTORC1 inhibition decreased CDK4 expression in human monocyte-derived macrophages (Supplementary Fig. 5f). Inhibition of CDK4 activity with the CDK4/6 inhibitors PD-0332991 or SC-20387331 potently blocked cell cycling in CSF1-stimulated $T s c 2^{\text {fl/fl }}$ and $T_{s c} 2^{\mathrm{f} / / \mathrm{fl}}$ Lyz2-Cre macrophages but did not induce apoptosis in vitro (Fig. $4 \mathrm{e}$ and Supplementary Fig. 5g,h). Accordingly, PD-0332991 treatment of older Tsc $2^{\text {fl/fl } L y z 2-C r e}$ mice that had established granulomas did not result in a marked disappearance of granulomas (data not shown). However, when we initiated granuloma formation by 
transplanting $T s c 2^{\mathrm{fl} / \mathrm{fl}} L y z 2$-Cre bone marrow into wild type mice, inhibition of CDK4 with PD-0332991 was able to prevent granuloma induction and formation (Fig. 4f,g). Because inhibition of CDK4 with PD-0332991 blocked proliferation (Fig. 4e), but did not influence CDK4 expression itself (Figure 4h) we reasoned that CDK4 induction by CSF1 via mTORC1 is causative for cell proliferation. Collectively, these data show that the mTORC1 pathway induces the expression of CDK4 upon stimulation by CSF1 to drive proliferation and granuloma formation.

\section{TSC2 regulates metabolism via CDK4-dependent glycolysis}

Cell proliferation needs to be metabolically supported and mTORC1 has been shown to be a major regulator of cellular metabolism32. Because we observed the enrichment of genes involved in glycolysis and oxidative phosphorylation in TSC2-deficient macrophages (Figure 3b), we measured as their indicators the extracellular acidification rate (ECAR) and the oxygen consumption rate (OCR), respectively. Basal and LPS-induced ECAR and OCR were both increased in $T s c 2^{\mathrm{fl} / \mathrm{fl}} L y z 2$-Cre BMDM compared to controls (Fig. 5a and Supplementary Fig. 6a) indicating higher glycolysis and mitochondrial respiration under chronic mTORC1 activation in macrophages. Consistent with this mTORC1-dependent glucose uptake was significantly higher in BMDM and in peritoneal macrophages from $T_{s c} 2^{\mathrm{f} / / \mathrm{fl}}$ Lyz2-Cre mice (Fig. 5b,c). Absolute glucose levels were decreased in $T s c 2^{\mathrm{f} / / 1} L y z 2$ Cre BMDM and in the lung of $T s c 2^{\text {fl/fl }} L y z 2$-Cre animals (Fig. $5 \mathrm{~d}$ and Supplementary Fig. 6b). Consistent with the higher OCR, constitutive mTORC1 activation increased mitochondrial mass in the cells (Fig. 5e,f); an effect that was reversed by rapamycin (Fig. 5e). A mitochondrial stress test indicated higher mitochondrial spare respiratory capacity of the $T s c 2^{\mathrm{fl} / \mathrm{fl}}$ Lyz2-Cre mitochondria (Supplementary Fig. 6c). This high mitochondrial respiration activity was in agreement with reduced citric acid levels found in $T s c 2^{\mathrm{fl} / \mathrm{fl}} L y z 2$ Cre BMDM (Supplementary Fig. 6d). Next, we aimed to determine whether CDK4 contributed to the TSC2-dependent reprogramming of the cellular metabolism. Indeed, the CDK4 inhibitor PD-0332991 significantly inhibited ECAR in Tsc2 ${ }^{\mathrm{fl} / \mathrm{fl}} L y z 2$-Cre BMDM (Fig. 5g). Moreover, inhibition of CDK4 reduced CSF1-stimulated ECAR activity in $T s c 2^{\mathrm{fl} / \mathrm{fl}} \mathrm{BMDM}$, while it did not influence glycolysis in CSF1-deprived cells (Fig 5h) suggesting that CSF1-induced activation of CDK4 via TSC2 contributes to glycolysis in macrophages. We wanted to corroborate these metabolic results in situ by measuring the enzymatic activities of glyceraldehyde 3-phosphate dehydrogenase (GAPDH), lactate dehydrogenase (LDH), isocitrate dehydrogenase (IDH), and succinate dehydrogenase (SDH) on frozen tissue sections. We found in the lungs of $T s c 2^{\text {fl/fl }} L y z 2$-Cre mice significantly more p-S6-positive macrophages with high activities for GAPDH, IDH and SDH than in the lungs of control animals, while the amount of cells with high LDH activity was similar (Fig. 5i,j). Similarly, we observed more p-S6-positive macrophages with high GAPDH activity in the liver of $T s c 2^{\mathrm{fl} / \mathrm{fl}} L y z 2$-Cre mice (Supplementary Fig. 6e,f). To identify the cellular functions that are fueled by the enhanced glucose metabolism in $\mathrm{T} c 2^{\mathrm{fl} / \mathrm{fl}} L y z 2$-Cre macrophages, we inhibited glycolysis with 2-deoxy-D-glucose (2-DG). 2-DG at doses of only $0.25 \mathrm{mM}$

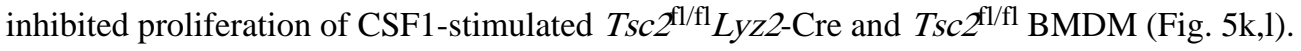
Notably, 2-DG induced cleavage of caspase-3 in CSF1-deprived $T_{s c 2^{\mathrm{fl} / \mathrm{fl}}}$ and in $T_{s c} 2^{\mathrm{fl} / \mathrm{fl}}$ Lyz2-Cre BMDM, but was unable to do so in CSF1-stimulated macrophages (Fig. $5 \mathrm{~m})$. These results show that deletion of TSC2 rewires the metabolism of macrophages 
towards increased CDK4-dependent glycolysis and mitochondrial respiration that is critical for proliferation but also inhibits apoptosis under growth factor-deprived conditions.

\section{Granulomas in human sarcoidosis show active mTORC1 signaling}

The observations in $T s c 2^{\mathrm{fl} / \mathrm{fl}} L y z 2$-Cre mice most strongly resemble the histological phenotype of patients with sarcoidosis; e.g. alveolar-like epithelioid macrophages that accumulate in the interstitium and express high levels of Lgals3. This prompted us to analyze whether mTORC1 activation is found in biopsies of sarcoidosis patients. We noticed activated mTORC1 signaling in granulomatous lesions in $33 \%$ out of 27 biopsies in an initial screening (Fig. 6a) with the hypertrophic cells being also positive for Mac-2 (Fig. 6b). To substantiate these findings, we reanalyzed a published clinical study, which compared active but self-limiting with active but progressive sarcoidosis in 15 patients9. We performed GSEA for hallmark gene sets and found the mTORC1 pathway significantly enriched (FDR $<0.001$ ) in the progressive set (Fig. 6c). E2F targets (FDR < 0.001) and a glycolysis gene set (FDR < 0.036) were also enriched in this patient population suggesting that macrophage proliferation and glycolysis contribute to the severity of the disease (Fig. 6c). While TSC2 was expressed at comparable levels, mRNA expression of $T S C 1$ was significantly decreased in patients with the progressive relative to the self-limiting form of the disease (Supplementary Figure 7). Strikingly, the gene set that was differentially expressed between the self-limiting and the progressive disease separated $T s c 2^{\mathrm{fl} / \mathrm{fl}}$ and $T s c 2^{\mathrm{fl} / \mathrm{fl}} L y z 2$-Cre BMDM in an unsupervised cluster analysis of the mRNA gene expression data (Fig. 6d). Moreover, we detected active cell proliferation inside the granulomas in $44 \%$ of 27 human sarcoidosis patients (Fig. 6e,f). A positive association ( $\mathrm{p}=0.003$ ) of $\mathrm{p}-\mathrm{S} 6$ and $\mathrm{Ki}-67$ in the granulomas of these patients confirmed the significance of that finding (Fig. $6 \mathrm{~g}$ ). Taken together, these results suggest that mTORC1-dependent macrophage proliferation and glycolysis contribute to disease progression in sarcoidosis.

\section{mTORC1 inhibition resolves granulomas in mice}

The link of mTORC1 with sarcoidosis progression encouraged us to study whether the granuloma phenotype in $T s c 2^{\mathrm{fl} / \mathrm{fl}}$ Lyz2-Cre mice was dependent on constitutive mTORC1 activation. After a treatment period of three weeks with the mTORC1 inhibitor everolimus33, the granulomas completely resolved in 8-week-old as well as 20 week-old $T s c 2^{f 1 / f 1} L y z 2$-Cre mice and we observed a normal lung architecture, whereas in placebotreated $T_{s c} 2^{\mathrm{f} / \mathrm{fl}} L y z 2$-Cre mice granulomas were well visible (Fig. 7a,b). Moreover, the swelling of the extremities also disappeared upon two weeks of mTORC1 inhibition in the old mice in vivo (Fig. 7c,d). Mechanistically, the hypertrophic macrophages downregulated S6 phosphorylation and underwent rapid apoptosis already two days after everolimus treatment (Fig. 7e). These results led us to conclude that the development and maintenance of granulomas initiated by hypertrophic macrophages in $T_{s c} 2^{\mathrm{fl} / \mathrm{fl}} \mathrm{Lyz2}$-Cre mice depends on the constitutive activation of mTORC1 and its inhibition restores normal tissue homeostasis in these mice. 


\section{Discussion}

In this work we propose that mTORC1 is a critical rheostat that negatively regulates macrophage quiescence, whereas its constitutive activation is sufficient to initiate granuloma formation in various tissues of mice. Moreover, we present evidence that activation of mTORC1 and macrophage proliferation determines the switch from a self-limiting to a progressive form in the human granulomatous disease sarcoidosis.

The induction and maintenance of granulomas remains poorly understood despite their importance for modifying disease pathology 11 . For example, excessive granuloma formation can be detrimental in non-infectious granulomatous disorders such sarcoidosis or Crohn's disease but can also favor pathogen growth and survival in tuberculosis and schistosomiasis 3,11 . Although we understand the morphological sequence of events during granuloma formation 1,3, molecular knowledge about signaling pathways that transform macrophages into hypertrophic, epithelioid macrophages that induce granulomas is virtually nonexistent. Unexpectedly, we found that myeloid-specific deletion of $T s c 2$ to activate mTORC1 was sufficient to initiate and maintain granuloma formation by enhancing macrophage proliferation and inhibition of apoptosis in vivo. Importantly, activation of mTORC1 already promoted the transformation of BMDM into hyperproliferative and hypertrophic, epithelioid cells that formed granulomatous structures in vitro. These results suggest granuloma formation as an intrinsic feature of macrophages. Currently, a unifying paradigm is missing from the explanation of why diverse pathogens, cytokines, nondegradable particles, or genetic disorders initiate the formation of granulomas. Our results suggest a model, in which the inability to clear a stimulus initiates and maintains mTORC1 activity to induce cell hypertrophy and granuloma formation. Therefore, the $T_{s c} 2^{\mathrm{fl} / \mathrm{fl}} L y z 2$ Cre mice present a potentially useful tool to study the molecular principles of granuloma formation in non-infectious and infectious diseases in vivo, and allow dissection of cellintrinsic pathways necessary for granuloma initiation from pathogenic signals that modify granuloma organization.

Macrophage proliferation was already described in sarcoidosis and other human granulomatous diseases34,35. Moreover, macrophages also proliferate in experimental granulomatous models36,37, however, the contribution of macrophage proliferation itself to granuloma formation has not been investigated mechanistically. Our data indicate that mTORC1-dependent macrophage proliferation contributes mainly to the establishment of the granulomatous phenotype in $\mathrm{Tsc} 2^{\mathrm{fl} / / \mathrm{fl}} L y z 2$-Cre mice. There is a renewed interest in defining the mechanisms of macrophage proliferation in vivo during pathologies such as atherosclerosis or obesity and under steady-state conditions to maintain the macrophage pool in tissues38-40. Our gene expression analysis in BMDM suggests that cell cycle progression and E2F transcription factors are the main targets of TSC2-mTORC1 in macrophages, a finding that was unexpected despite the knowledge of the role of mTORC1 for cell proliferation in other cells41,42. An important finding from our study is that CDK4 expression is induced by the growth factor CSF1 via TSC2-mTORC1 to promote macrophage proliferation. Current belief suggests that CDKs remain largely constant throughout the cell cycle30, however, we show that the cell cycle is not only controlled by the amount of cyclins but also by the expression of a CDK. It can be speculated that the 
presence of adequate amounts of CDKs such as CDK4 is rate limiting in addition to sufficient amounts of cyclins. In line with this suggestion, transgenic expression of CDK4 in the epidermis is sufficient to drive cell cycle progression as well as hypertrophy43 similar to

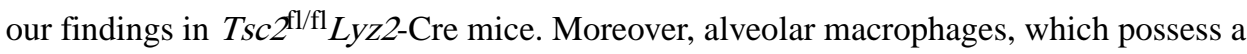
high self-renewal proliferative capacity, also express high levels of CDK4. While we found enhanced proliferation, we also observed the upregulation of survival genes and decreased apoptosis in BMDM in vitro and in Tsc $2^{\mathrm{fl} / / \mathrm{l}}$ Lyz2-Cre mice in vivo. This contributed to the maintenance of the granulomas, as short time inhibition of mTORC1 readily induced apoptosis in the macrophages in vivo and completely resolved the granulomas. Hence, TSC2-mTORC1 tunes a balance between proliferation and apoptosis that is important for macrophage tissue homeostasis.

Transitions between quiescent and activated or proliferating states require the reprogramming of the cellular metabolism to support these functional changes 44,45 . We observed a prominent remodeling of the cellular metabolism towards increased glycolysis as well as oxidative phosphorylation in $T_{s c} 2^{\mathrm{fl} / \mathrm{fl}} L y z 2$-Cre macrophages by enhancing GAPDH, IDH, and SDH activities that supported macrophage proliferation. Limiting the glycolytic flux radically suppressed cell cycling and proliferation corroborating the important role of glucose as carbon source for macrophages45. We describe a novel role of mTORC1 for regulating the cellular metabolism 21 and show that CDK4 contributes to mTORC1dependent glycolysis to fuel proliferation.

Sarcoidosis onset is gradual from an asymptomatic state to a progressive disease that can become life threatening 9. Interestingly, a genome-wide association study identified 12q13.3-q14.1 as a risk locus for sarcoidosis that induces overexpression of eight genes including CDK4 in four different populations46 and macrophage proliferation as well as decreased apoptosis were observed in sarcoidosis35. Moreover, sarcoid granulomas are known to have a high uptake capacity for glucose 47 strengthening the observed association of mTORC1 activation and glycolysis with sarcoidosis progression. While we found decreased expression of Tsc1 in progressive sarcoidosis patients, the underlying reason why these patients have active mTORC1 signaling as our study suggests is currently unclear. Somatic mutations affecting the mTOR pathway or environmental triggers that the immune system is unable to clear could both promote mTORC1 activation to drive sarcoidosis progression. Hypercalcemia is diagnosed in about $5-10 \%$ of the patients; however, blood calcium levels were similar in 11-week old $T s c 2^{\mathrm{fl} / \mathrm{fl}}$ and $T s c 2^{\mathrm{fl} / / \mathrm{fl}} L y z 2$-Cre mice (data not shown). For treatment of sarcoidosis corticosteroids are usually recommended, but they are associated with significant side effects5,7. Moreover, some patients are refractory to treatment and there is a significant rate of relapse5,48. Our findings point to the evaluation of mTOR inhibitors in this patient group. Indeed, successful treatment of a sarcoidosis patient with the mTOR inhibitor rapamycin has been reported49. Finally, our results warrant the assessment of whether mTORC1-dependent macrophage proliferation and granuloma formation contributes to disease outcomes in other non-infectious and infectious granulomatous diseases. 


\section{Methods}

\section{Mouse strains}

B6;129-Tsc $2^{\text {fl/fl }}$ mice50 were crossed to $L y z 2^{\text {cre/+ }}$ mice to obtain $T s c 2^{\mathrm{fl} / \mathrm{fl}} L y z 2^{\text {cre/++ }}$ (denoted $T s c 2^{\mathrm{fl} / \mathrm{fl}} L y z 2$-Cre) or $T s c 2^{\mathrm{fl} / \mathrm{fl}} L y z 2^{+/+}$(denoted $T s c 2^{\mathrm{fl} / \mathrm{fl}}$ ) littermates. Additionally, $T s c 2^{1 \mathrm{l} / \mathrm{fl}} \mathrm{Lyz2}$-Cre mice backcrossed to a pure C57BL/6 background by speed congenics retained the observed granulomatous phenotype (data not shown). All mouse studies were approved by the official Austrian ethics committee for animal experiments (GZ.BMWF-66.009/0304-II/3b/2013 and GZ.BMWF-66.009/0116/II/3b/2014). Male and female mice were used randomly and no major sex-specific differences were observed. Groups in individual experiments were sex-matched and age-matched.

\section{Treatment with everolimus}

8 and 20 week-old $T s c 2^{\mathrm{fl} / \mathrm{fl}} \operatorname{Lyz2}$-Cre mice were gavaged with $5 \mathrm{mg} / \mathrm{kg}$ body weight everolimus or provided placebo daily for three weeks. Short time treatment lasted for two days, whereas half the dose everolimus or placebo was administered in the morning, the other half in the evening. Everolimus and placebo were dissolved in mouse drinking water. Everolimus (as 2\% microemulsion) and placebo were provided by Novartis.

\section{Bone marrow transplantation}

4 week-old female C57BL/6;129 mice were lethally irradiated with 9.5 Gy using a XYLON Maxishot (YXLON International GmbH, Hamburg, Germany). The next day they received $1 \times 10^{6}$ mononuclear bone marrow cells from male $T s c 2^{\mathrm{fl} / \mathrm{fl}}$ or $T s c 2^{\mathrm{fl} / \mathrm{fl}} L y z 2$-Cre mice into the tail vein. Phenotypical analysis was performed three months after BMT.

\section{Treatment with Palbociclib (PD-0332991)}

Bone marrow transplantation with $T s c 2^{\mathrm{fl} / \mathrm{fl}} \mathrm{Lyz} 2$-Cre bone marrow into C57BL/6;129 mice was performed as above. The mice were left to recover for 10 days, whereafter they were gavaged daily with $100 \mathrm{mg} / \mathrm{kg}$ PD-0332991 (Chemitek) or solvent control for two month. PD-0332991 was dissolved in PBS $(15 \mathrm{mg} / \mathrm{ml})$ aliquoted and stored at $-80^{\circ} \mathrm{C}$. Fresh aliquots of inhibitor or PBS were defrosted each day and sodium lactate (Sigma) was added to obtain a final concentration of $50 \mathrm{mM}$ sodium lactate in either inhibitor or solvent control.

\section{Cell culture}

Cells were cultured at $37^{\circ} \mathrm{C}$ in a humidified $\mathrm{CO} 2(5 \%)$ incubator. Bone marrow was isolated from femur, tibia and humerus and differentiated for 6 days on petri dishes (for the whole bone marrow 6 plates were used containing each 10-12 ml) in 10-20\% L929 supplemented 10\% MФ media: DMEM high glucose (Gibco), 10\% low endotoxin FBS (Gibco), 2 mM Lglutamine (Lonza), $100 \mathrm{U} / \mathrm{ml}$ penicillin (Sigma), $100 \mu \mathrm{g} / \mathrm{ml}$ streptomycin (Sigma), $50 \mu \mathrm{g} / \mathrm{ml}$ $\beta$-mercaptoethanol (Gibco). Three days later floating cells were removed and adherent cells were splitted 1:2 in fresh L929 supplemented media. Differentiated BMDM ( $296 \%$ positive for F4/80 and CD11b) were seeded in 10\% MФ media (without L929 supplementation) and were let to recover for 4 hours. BMDM were pre-inhibited for 1 hour before overnight stimulation, or treated as indicated. For dendritic cell differentiation bone marrow was 
differentiated for 6 days on cell culture treated dishes (one 6-well plate per mouse and $4 \mathrm{ml}$ per well) in DC-media: RPMI 1640 (Lonza) 10\% low endotoxin FBS (Gibco), 2 mM Lglutamine (Lonza), $100 \mathrm{U} / \mathrm{ml}$ penicillin (Sigma), $100 \mu \mathrm{g} / \mathrm{ml}$ streptomycin (Sigma), $50 \mu \mathrm{g} / \mathrm{ml}$ $\beta$-mercaptoethanol (Gibco) supplemented with $20 \mathrm{ng} / \mathrm{ml} \mathrm{CSF} 2$. Two days later half the medium was replaced and the next day floating cells were removed carefully and fresh medium was added. Differentiated DCs (floating cell portion; $292 \%$ positive for CD11c and CD11b were re-seeded in CSF2 supplemented DC-media. Human PBMCs were isolated using standard picoll gradients, floating cells were removed and monocytes were cultured for 7 days in RPMI 1640 (Lonza) containing 10\% FBS (Gibco), $100 \mathrm{U} / \mathrm{ml}$ penicillin (Sigma), $100 \mu \mathrm{g} / \mathrm{ml}$ streptomycin (Sigma). Inhibitors or stimuli were used in following concentrations, except otherwise indicated; Rapamycin $100 \mathrm{nM}$ (Calbiochem), Torin 250 nM (Tocris), Palbociclib (PD-0332991) HCl $1 \mu \mathrm{M}$ (Selleckchem), 2-Bromo-12,13dihydro-5H-indolo[2,3-a]pyrrolo[3,4-c]carbazole-5,7(6H)-dione 0.5-1 $\mu \mathrm{M}$ (Santa Cruz), murine CSF1 $10 \mathrm{ng} / \mathrm{ml}$ (Peprotech), human CSF1 20 ng/ml (Peprotech), IL-4 10 ng/ml (Peprotech), LPS (E. coli 0111.B4) 100 ng/ml (Sigma), IFN $\gamma 20$ ng/ml (Peprotech), murine CSF2 20 ng/ml (Peprotech).

\section{siRNA in fibroblasts}

CCD1079Sk were obtained from ATCC (CRL-2097); IMR-90 were bought from ATCC (CCL-186); both cell lines were STR profiled. $T s c 1^{+/+}$MEFs were a kind gift of D.J. Kwiatkowski. All cell lines were tested negative for mycoplasma contamination. Primary human foreskin CCD1079Sk and lung IMR-90 fibroblasts, as well as an immortalized mouse embryonic fibroblast cell line were maintained on tissue culture-treated plates in Dulbecco's Modified Eagle Medium (DMEM) at $4.5 \mathrm{~g} / \mathrm{L}$ glucose (Invitrogen) supplemented with $10 \%$ fetal calf serum (Sigma) and $2 \mathrm{mM} \mathrm{L-Glutamine.} \mathrm{siRNA} \mathrm{transfection} \mathrm{of} \mathrm{primary}$ and immortalized fibroblasts was performed using Lipofectamine RNAiMAX (Invitrogen) as described previously51. Pooled siRNAs specifically targeting human TSC2 or mouse Tsc2 (Dharmacon, ON-TARGETplus SMART pool reagents) were delivered to the cells at a final concentration of $50 \mathrm{nM}$. A pool of four non-targeting siRNAs was used as a control for non-sequence-specific effects. Non-treated cells were co-analyzed to monitor non-specific effects due to the transfection procedure. 72 hours after the knockdown the MEFs were analyzed under the microscope and harvested for immunoblotting, whereas CCD1079Sk and IMR-90 were reseeded for 60 hours, serum-starved for 12 hours before analysis.

\section{Proliferation assay}

For CellTiter-Blue analysis, $1.8 \times 10^{3}$ cells were seeded in 96-well plates. 4 hours later they were inhibited as indicated for 60 minutes and thereafter stimulated with $40 \mathrm{ng} / \mathrm{ml} \mathrm{CSF} 1$. At the indicated time points CellTiter-Blue ${ }^{\circledR}$ (Promega) was added 1:11 and cells were further incubated for 2 hours at $37^{\circ} \mathrm{C}$. Fluorescence intensity was measured at a $530 \mathrm{~nm}$ excitation wavelength and a $590 \mathrm{~nm}$ emission wavelength using a Synergy HT Photometer (Biotek Instruments, Winooski, VT). A replicate plate was used for each time point.

\section{Flow Cytometry}

BMDM were harvested and suspended in FACS buffer (PBS with 2\% FBS and $2 \mathrm{mM}$ EDTA). Spleens were forced through a $70 \mu \mathrm{m}$ cell strainer and red blood cells were lysed 
with ACK lysis buffer $\left(\mathrm{NH}_{4} \mathrm{Cl} 0.15 \mathrm{M}, \mathrm{KHCO}_{3} 10 \mathrm{mM}\right.$, EDTA $\left.0.1 \mathrm{mM}\right)$. Perfused lungs were chopped in small pieces and digested at $37^{\circ} \mathrm{C}$ for 1 hour in RPMI 1640 (Lonza) containing 11.5\% HBSS (Gibco) 4.4\% FBS (Gibco), 1 mg/ml Collagenase Type I (Gibco) and $50 \mathrm{U} / \mathrm{ml}$ DNase I (Invitrogen). Tissue was forced through a $70 \mu \mathrm{m}$ cell strainer, thereafter red blood cells were lysed and the flow-through of a $40 \mu \mathrm{m}$ cell strainer was collected in FACS buffer. For surface marker staining cell suspensions were blocked with TruStain fcX ${ }^{\mathrm{TM}}$ (Biolegend) for 20 minutes and stained light protected for 25 minutes on ice with the indicated antibodies. Pellets were washed and suspended in fresh FACS buffer prior analysis. Antibodies from Biolegend were F4/80 (BM8) 1:100, CD11b (M1/70) 1:100-200, CD11c (N418) 1:100, Gr-1 (RB-68C5) 1:100, CD64 (X54-5/7.1) 1:50, CD45.2 (104) 1:100, Mac-2 (M3/38) 1:100, CD24 (M1/69) 1:50, CD4 (RM4-5) 1:100, CD8a (53-5.7) 1:100 and I-A $^{\mathrm{b}}$ (AF6-120.1) 1:100. CD11b (M1/70) 1:100-200, CD11c (N418) 1:100, CD45.2 (104) 1:100, 1:100 CD3e (eBio500A2) and CD115 (AFS98) 1:100 were obtained from eBioscience and Ly-6C (AL-21) 1:200, CD19 (1D3) 1:100 and Siglec-F (E50-2440) 1:100 were purchased from BD. Data were obtained on a FACS Calibur instrument or FACSCanto II (both BD Biosciences) and processed using FlowJo (Tree Star Inc, Ashland, OR). Gating for myeloid subpopulations in the lung was performed as previously described27. T-cells were defined as $\mathrm{CD} 45.2+\mathrm{CD} 3 \mathrm{e}+$ and positive for $\mathrm{CD} 4$ or $\mathrm{CD} 8$ respectively, B-cells were defined as CD45.2+ CD19+ $\mathrm{IA}^{\mathrm{b}}+$.

\section{Cell cycle analysis}

For cell cycle analysis, $150 \mu \mathrm{l}$ of cell suspension was added dropwise (while vortexing) to $750 \mu \mathrm{l} 85 \% \mathrm{EtOH}$ (to obtain a final concentration of $70 \% \mathrm{EtOH}$ for fixation) and frozen at $-20^{\circ} \mathrm{C}$ for at least one night. Peritoneal lavage cells, after erythrocyte lysis, were stained with CD115 before fixation. The pellet was suspended in $300 \mu \mathrm{l}$ PI-staining solution $(50 \mu \mathrm{g} / \mathrm{ml}$ Propidium iodide, $250 \mu \mathrm{g} / \mathrm{ml}$ RNase A, $400 \mathrm{nM}$ trisodium citrate dehydrate, $0.1 \%$ TritonX-100), incubated for light protected for 20 minutes on ice and resuspended in PBS. Cells were analyzed on a FACS Calibur (BD Biosciences).

Alternatively, $1 \mu \mathrm{M}$ EdU (Invitrogen, Click-iT ${ }^{\circledR}$ EdU Flow Cytometry Assay) was added to the culture medium and cells were replaced in the incubator. Two hours later the cells were washed with $1 \%$ BSA-PBS and harvested. The pellets where resuspended in cold $\left(-20^{\circ} \mathrm{C}\right)$ $\mathrm{MeOH}$ and incubated for 10 minutes at $-20^{\circ} \mathrm{C}$, whereafter $\mathrm{MeOH}$ was discarded. Cells were washed and $125 \mu \mathrm{l} \mathrm{Click-i \textrm {T } ^ { \circledR }}{ }^{\circledR}$ reaction cocktail was added to each sample. After $30 \mathrm{~min}$ (at room temperature and light protected) the pellets were washed again with $1 \%$ BSA-PBS and resuspended in $125 \mu \mathrm{l}$ RNaseA/7-AAD ( $8 \mu \mathrm{g} / \mathrm{ml}$ and $400 \mu \mathrm{g} / \mathrm{ml}$ respectively). After a further light protected $30 \mathrm{~min}$ incubation at room temperature the supernatant was discarded and resuspended in FACS buffer. Cells were analyzed on a FACS Calibur (BD Bioscience).

\section{Glucose uptake}

For 2-NBDG uptake, BMDM were harvested, suspended in PBS and incubated with $100 \mu \mathrm{M}$ 2-NBDG (Life Technologies) for 10 minutes at $37^{\circ} \mathrm{C}$. Peritoneal lavage, after erythrocyte lysis, was stained with F4/80 before 2-NBDG addition. Cells were washed and analyzed on a FACS Calibur (BD Biosciences). 


\section{Mitochondrial staining}

For staining of mitochondria, $60 \mathrm{nM}$ of MitoTracker ${ }^{\circledR}$ Green FM (ILife Technologies) was added to the cell culture medium for 30min, thereafter cells were harvested and analyzed by flow cytometry. Cells were analyzed on a FACS Calibur (BD Biosciences).

\section{Immunoblotting}

Cells were washed with ice cold PBS, scraped in PBS and the pellet was dissolved in lysis buffer (20 mM Hepes pH 7.9, $0.4 \mathrm{M} \mathrm{NaCl}, 25 \%$ (v/v) Glycerin, $1 \mathrm{mM}$ EDTA, $0.5 \mathrm{mM}$ $\mathrm{Na}_{3} \mathrm{VO}_{4}, 0.5 \mathrm{mM}$ DTT, $1 \%$ Triton X-100) supplemented with protease and phosphatase inhibitors (Roche) and $4 \mu \mathrm{g} / \mathrm{ml}$ aprotinin, $4 \mu \mathrm{g} / \mathrm{ml}$ leupeptin, $0.6 \mu \mathrm{g} / \mathrm{ml}$ benzamidinchloride, $20 \mu \mathrm{g} / \mathrm{ml}$ trypsin inhibitor and $2 \mathrm{mM}$ PMSF (all from Sigma). Protein concentration was measured and equal amounts of denaturated lysate were resolved on 7.5-12\% SDS-PAGE and transferred to nitrocellulose membranes. Membranes were blocked in $4 \%$ low-fat milk for 1 hour at room temperature and incubated with primary antibodies at $4^{\circ} \mathrm{C}$ overnight. HRP-conjugated secondary antibodies (Bethyl) 1:10000 were applied for 1 hour at room temperature in $4 \%$ low-fat milk and proteins were visualized using ECL substrate (Thermo Scientific).

Primary antibodies were TSC2 (D93F12) 1:1000, p-Akt S473 (D9E) 1:1000, 4E-BP1 (53H11) 1:1000, p-S6 S240/244 (rabbit polyclonal) 1:3000, S6 (54D2) 1:1000, p-Akt T308 (D25E6) 1:1000, pan-Akt (40D4) 1:1000, $\beta$-tubulin (9F3) 1:1000 , p-RB Ser807/811 (D20B12) 1:1000, PDCD4 (D29C6) 1:1000, cleaved caspase 3 Asp175 (5A1E or rabbit polyclonal) 1:500, survivin (71G4B7) 1:1000 and p-S6K1 T389 (108D2) 1:1000, p-TSC2 S939 (rabbit polyclonal) 1:1000 obtained from Cell Signaling Technology. Bcl-2 (N-19) 1:500, cyclin D1 (M-20) 1:500, p27Kip1 (C-19) 1:1000, cyclin A (C-19) 1:500, CDK4 (C-22) 1:1000, CDK6 (H-96) 1:500, and E2F1 (KH95) 1:500 were fom Santa Cruz. atubulin (CP06) 1:3000 was purchased from Calbiochem and GAPDH (rabbit polyclonal) 1:10000 from Trevigen.

\section{Immunofluorescence microscopy}

Cells were seeded overnight in 8-well PCA Chamber slides (Sarsteadt). Cells were washed, fixed for 10 minutes with $4 \%$ paraformaldehyde at room temperature and rinsed with PBS. Exess PFA was quenched for 15 minutes with $100 \mathrm{mM}$ glycine wherafter slides were incubated for 30 minutes in PBS. Cell were then permeabilized for 7 minutes with $0.5 \%$ TritonX wereafter slides were rinsed and then incubated for 30 minutes in PBS. Blocking was performed for 1 hour with $1 \%$ BSA and after excessive washing the slides were stained with p-S6 S240/244 (rabbit polyclonal) from CST and ATPB (3D5) from Abcam overnight at $4{ }^{\circ} \mathrm{C}$. The next day were rinsed and then incubated for $3 \times 20$ minutes in PBS. Cells were stained with A546-labeled secondary antibodies (Invitrogen) 1:500 rinsed and then incubated for 2x20 minutes in PBS. Nuclear tracking was performed for 5 min using 0.1 $\mathrm{mg} / \mathrm{ml}$ Hoechst-33342 (Invitrogen) where after slides were washes extensively and mounted in Vectashield mounting medium. 


\section{mRNA expression analysis}

Shock-frozen lung tissue was homogenized in TRI Reagent ${ }^{\circledR}$ in a Precellys homogenizer. BMDM were washed and suspended directly in TRI Reagent ${ }^{\circledR}$. RNA was isolated according to the manufactures instruction. Equal amounts of RNA were converted to cDNA using GoScriptTM Reverse Transcription system (Promega). mRNA levels were determined by using GoTaq ${ }^{\circledR}$ qPCR Master Mix (Promega) on a StepOnePlus ${ }^{\mathrm{TM}}$ Real-Time PCR System. Relative expression was normalized to $\beta$-actin. The following primers pairs were used: Chil3, CCAGCAGAAGCTCTCCAGAAGCA, TGGTAGGAAGATCCCAGCTGTACG; Retnla, CTGCCCTGCTGGGATGACTGCTA, AGCGGGCAGTGGTCCAGTCAA; Igfl, ATCTGCCTCTGTGACTTCTTGA, GCCTGTGGGCTTGTTGAAGT; II10, CCCCAGGCAGAGAAGCATGGC, GGGGAGAAATCGATGACAGCGCC; $\mathrm{Mrcl}$, ACATGCCAGGACGAAAGGCGG, TTGTGGGCTCTGGTGGGCGA; Lgals3, GGCAGACAGCTTTTCGCTTA, GATAAGCAGCCCCTGGGTAG; Itgam, CATCAAGGGCAGCCAGATTG, GAGGCAAGGGACACACTGAC; $\mathrm{Ccl2}$, AGCTGTAGTTTTTGTCACCAAGC, GTGCTGAAGACCTTAGGGCA; II12b, GCAGCGTGGGAGTGGGATGTG, GGGCATCGGGAGTCCAGTCCA; iNOS, TGGCAACATCAGGTCGGCCAT, CGGGCATCTGGTAGCCAGCG; Actb, TTGCACATGCCGGAGCCGTT, CACACCCGCCACCAGTTCGC.

\section{Microarray analysis}

BMDM ( $\mathrm{n}=4$ per group) were seeded for 18 hours in $10 \%$ MФ media. Quiagen RNeasy Plus Micro Kit was used to isolate total RNA according to manufactures instructions. Total RNA was subjected to microarray analysis on Affymetrix GeneChip ${ }^{\circledR}$ Mouse Gene 2.0 ST arrays. Normalization of the data was done with the Affymetrix Expression Console. Analysis was performed with the Affymetrix Transcriptome Analysis Console (TAC) v3.0. We considered genes to be differentially regulated between $T s c 2^{\mathrm{fl} / f 1}$ and $T s c 2^{\mathrm{fl} / \mathrm{fl}} L y z 2$-Cre BMDM that were more than two-fold differently expressed with a ANOVA significance value of smaller than 0.05. Microarray data have been deposited under GEO accession number GSE77075.

\section{Gene set enrichment analysis (GSEA)}

For the identification of enriched gene signatures, we used the gene set enrichment analysis (GSEA) tool available from the Broad Institute website. GSEA was performed by comparison of normalized gene-expression data obtained from $T s c 2^{\mathrm{fl} / \mathrm{fl}}$ and $T s c 2^{\mathrm{fl} / / \mathrm{f}} L y z 2$ Cre BMDM ( $\mathrm{n}=4$ /group). We used 1,000 gene-set permutations for testing of significance. We screened the collection of signatures under the categories $\mathrm{H}$ (hallmark gene sets) and C3.TFT (transcription factor targets). In addition, we used the KEGG-APOPTOSIS and selfrenewal set ("WONG_EMBRYONIC_STEM_CELL_CORE") from the Broad Institute website.

\section{Extracellular flux analysis}

Cells were seeded in XF24 plates in 10\% M $\Phi$ media. The next day, cells were washed and ECAR and OCR were measured in $4.5 \mathrm{~g} / \mathrm{L}$ glucose-supplemented media in an XF24 Extracellular Flux Analyzer (Seahorse Bioscience). Results were normalized to the actual cell count after measurements. 


\section{Metabolic imaging of enzymatic activities}

This method allows the characterization of particular cells types and the simultaneous determination of their intracellular enzyme activities in structurally intact tissue (A. Haschemi, personal communication). Briefly, tissues were harvested, cut in $10 \mathrm{~mm}^{2}$ pieces, snap-frozen in O.C.T. (Tissue-Tek, Sakura) and stored at $-80^{\circ} \mathrm{C}$. Tissue sections were incubated in GAPDH, LDH, IDH or SDH activity assay medium and enzyme reactions were carried out for $15 \mathrm{~min}$ as previously reported (Noorden, CJFv, Frederiks, WM. Enzyme histochemistry: a laboratory manual of current methods. Oxford; New York Oxford: Oxford University Press; Royal Microscopical Society; 1992. vi, 116 p. p.). Slides were washed in PBS to stop the reaction. Tissue sections were further used for antibody stainings after blocking for $30 \mathrm{~min}$ with $3 \% \mathrm{BSA}$ and $0.01 \%$ Triton X-100. Sections were then either stained with directly labelled antibodies or no antibodies to control for background staining. Next, slides were washed and stained with DAPI and mounted in Mowiol 4-88. We used the following directly conjugated antibodies: Mac-2 (M3/38) Alexa Fluor 647 (Biolegend) and p-S6 (S240/244) Alexa 594 (Cell Signaling Technology). TissueFAXS acquisition was performed using a Zeiss Observer Z1 microscope equipped with a stage for 12-slides, a Hamamatsu Photonics Orca flash 4.0 Camera and a X-Cite Series 120PCQ Laser (TissueGnostics). All images were acquired using the TissueFAXS 4.0 Slides software by TissueGnostics and then analyzed by TissueQuest 4.0.1.0128 software (TissueGnostics). The analysis of DAPI was set to the default settings and cell detection parameters were set to a max exterior radius of $7 \mu \mathrm{m}$. The raw data, containing all relative intensities for each event (cell), was exported to Excel.

\section{Determination of metabolites in lung and BMDM}

$1 \mathrm{~mL}$ methanol/chloroform/water (M:C:W= 2.5:1:0.5) was added to frozen BMDM or lung tissue in a $2 \mathrm{ml}$ Precellys lysis kit tube. The samples were homogenized with $1 \mathrm{~mm}$ ceramic beads in a Precellys 24 homogenizer for 15 seconds at $5000 \mathrm{rpm}$ twice and centrifuged for 5 mins at $14000 \mathrm{x} \mathrm{g}$ and $4^{\circ} \mathrm{C}$ before the supernatant was transferred to an Eppendorf tube. The pellet was washed with $400 \mu \mathrm{MCW}$ by shortly vortexing and centrifuging it again before combining the wash supernatant with the first supernatant. Samples were dried in in a ScanSpeed MiniVac Beta at $35^{\circ} \mathrm{C}, 1100 \mathrm{rpm}$ and $0.0001 \mathrm{mbar}$ and subsequently frozen at $-80^{\circ} \mathrm{C}$. To induce a phase separation, samples were disolved in $500 \mu \mathrm{l} \mathrm{MCW}$ and $200 \mu \mathrm{l}$ milliQ $\mathrm{H}_{2} \mathrm{O}$, vortexed for 3 seconds and centrifuged at $14000 \mathrm{xg}$ for $5 \mathrm{~min}$ at $4^{\circ} \mathrm{C}$. The upper polar phase was transferred to a new Eppendorf tube and dried in the speedvac at $30^{\circ} \mathrm{C}, 650 \mathrm{rpm}$ and $0.0001 \mathrm{mbar}$. The polar fractions were incubated with Methoxaminhydrochloride $\left(30^{\circ} \mathrm{C} / 90\right.$ minutes) and subsequently with MSTFA (Methyl-N(trimethylsilyl)-trifluoroacetamide) $\left(37^{\circ} \mathrm{C} / 30\right.$ minutes $)$ to derivatize polar functional groups of the analytes before they were analyzed via GC(EI)MS. Areas of derivates belonging to one standard substance (confirmed by standard analysis and matching the spectra to the Golm Metabolome Database) were summed up and linear regressions were used to quantify the analytes in the samples. 


\section{Human sarcoidosis samples}

Pathological specimens of lung or mediastinal lymph nodes obtained from 27 patients with proven sarcoidosis were retrieved from the archives of the Department of Pathology, Medical University Vienna. The project was authorized by the Ethical Committee of the Medical University of Vienna (Ref. Nr. 194/2016) and was carried out in compliance with Austrian legislation. Diagnosis was based on radiological, clinical and histopathological criteria. Tissues were fixed in $4 \%$ formaldehyde and embedded in paraffin.

For the reanalysis of the study by Lockstone et a19, we downloaded the corresponding raw data from the NCBI GEO homepage (accession number GSE19976) and analyzed it with the Affymetrix Expression Console, the Affymetrix TAC v3.0, and the GSEA tool. The unsupervised cluster analysis of the microarray data of $T s c 2^{\mathrm{fl} / f \mathrm{l} l}$ and $T s c 2^{\mathrm{fl} / \mathrm{fl}} L y z 2-\mathrm{Cre}$ BMDM was performed with the genes that that were more than 1.5 -fold differently expressed in progressive relative to self-limiting sarcoidosis patients with an ANOVA significance value of smaller than 0.05 .

\section{Histology}

Mouse tissues were washed, fixed in $4 \%$ paraformaldehyde, dehydrated, embedded in paraffin and sectioned. Feet tissue was additionally decalcified with 15\% EDTA pH 7.2. Primary antibodies used for immunohistochemical stainings were CDK4 (C-22) from Santa Cruz, p-S6 S240/244 (rabbit polyclonal) and cleaved caspase 3 (5A1E) were purchased from CST. Ki-67 (H-300) was from Santa Cruz or from Abcam (ab66155), Mac-2 (M3/38) was obtained from Cederlane and MPO was from Dako. Biotinylated horse anti-mouse IgG or biotinylated horse anti-rabbit IgG were from Vector Laboratories. Novocastra streptavidinHRP (Leica) and AEC-high sensitivity substrate chromogen (Dako) were used for detection of primary antibodies. Alternatively VECTASTAIN Elite ABC Kit and DAB (Vector Laboratories) were utilized. For immunofluorescent staining of paraffin slides, primary antibodies used were p-S6 S240/244 from CST, Mac-2 (M3/38) either from Cederlane or directly labelled from Biolegend and F4/80 (CI:A3-1) from Serotec. Species-matched secondary antibodies (A488 and A546) were purchased from Invitrogen. Some images were quantitatively analyzed with ImageJ. Percentage of positive staining was determined by calculating the ratio between stained area and total tissue area within a predefined threshold. All pixels in the image with values below the threshold were counted.

\section{Statistical analysis}

Data were analyzed with GraphPad Prism (Version 6) or with Microsoft Excel. Data sets were compared with an unpaired two-tailed t-test and differences were considered significant if $\mathrm{P}$ values were $<0.05$. In this explorative study no statistical methods were used to predetermine sample size. Group sample size was chosen using records of variance in previous experiments. Mice or samples were randomly assigned to experimental groups or processing orders. The relationship of mTORC1 activation and proliferation in human sarcoidosis samples was evaluated in a blinded fashion and investigated using fisher's exact test. 


\section{Supplementary Material}

Refer to Web version on PubMed Central for supplementary material.

\section{Acknowledgments}

We thank U. Reichart and T. Kolbe for support in mouse breeding. We are grateful to D. Georg for the possibility of using the XYLON Maxishot. We would also like to thank M. Stadler and H. Dolznig for providing human monocyte-derived macrophages. T.W. is supported by grants from the Austrian Science Fund (FWF) grant FWFP27701-B20, the Else-Kröner-Fresenius-Stiftung (P2013_A149), and the Herzfelder'sche Familienstiftung. M.L. is supported by the [DOC] Doctoral Fellowship Programme of the Austrian Academy of Sciences. V.S. is funded by FWF SFB F28 and SBF F47; M.Mü. is funded by FWF SFB F28. M.Mi. is supported by the FWF grant P25336B13.

\section{References}

1. Cambier CJ, Falkow S, Ramakrishnan L. Host evasion and exploitation schemes of Mycobacterium tuberculosis. Cell. 2014; 159:1497-1509. [PubMed: 25525872]

2. Russell DG, Cardona PJ, Kim MJ, Allain S, Altare F. Foamy macrophages and the progression of the human tuberculosis granuloma. Nat Immunol. 2009; 10:943-948. [PubMed: 19692995]

3. Orme IM, Basaraba RJ. The formation of the granuloma in tuberculosis infection. Semin Immunol. 2014; 26:601-609. [PubMed: 25453231]

4. Hams E, Aviello G, Fallon PG. The schistosoma granuloma: friend or foe? Front Immunol. 2013; 4:89. [PubMed: 23596444]

5. Iannuzzi MC, Fontana JR. Sarcoidosis: clinical presentation, immunopathogenesis, and therapeutics. JAMA. 2011; 305:391-399. [PubMed: 21266686]

6. Petersen HJ, Smith AM. The role of the innate immune system in granulomatous disorders. Front Immunol. 2013; 4:120. [PubMed: 23745122]

7. Beegle SH, Barba K, Gobunsuy R, Judson MA. Current and emerging pharmacological treatments for sarcoidosis: a review. Drug Des Devel Ther. 2013; 7:325-338.

8. Zissel G, Muller-Quernheim J. Cellular Players in the Immunopathogenesis of Sarcoidosis. Clin Chest Med. 2015; 36:549-560. [PubMed: 26593132]

9. Lockstone HE, et al. Gene set analysis of lung samples provides insight into pathogenesis of progressive, fibrotic pulmonary sarcoidosis. Am J Respir Crit Care Med. 2010; 181:1367-1375. [PubMed: 20194811]

10. Ehlers S, Schaible UE. The granuloma in tuberculosis: dynamics of a host-pathogen collusion. Front Immunol. 2012; 3:411. [PubMed: 23308075]

11. Ramakrishnan L. Revisiting the role of the granuloma in tuberculosis. Nat Rev Immunol. 2012; 12:352-366. [PubMed: 22517424]

12. Orme IM, Robinson RT, Cooper AM. The balance between protective and pathogenic immune responses in the TB-infected lung. Nat Immunol. 2015; 16:57-63. [PubMed: 25521685]

13. Ehlers S, Kutsch S, Ehlers EM, Benini J, Pfeffer K. Lethal granuloma disintegration in mycobacteria-infected TNFRp55-/- mice is dependent on T cells and IL-12. J Immunol. 2000; 165:483-492. [PubMed: 10861087]

14. North RJ, Jung YJ. Immunity to tuberculosis. Annu Rev Immunol. 2004; 22:599-623. [PubMed: 15032590]

15. Regev D, et al. Heme oxygenase-1 promotes granuloma development and protects against dissemination of mycobacteria. Lab Invest. 2012; 92:1541-1552. [PubMed: 22964851]

16. Dorhoi A, Kaufmann SH. Pathology and immune reactivity: understanding multidimensionality in pulmonary tuberculosis. Semin Immunopathol. 2016; 38:153-166. [PubMed: 26438324]

17. Laplante M, Sabatini DM. mTOR signaling in growth control and disease. Cell. 2012; 149:274293. [PubMed: 22500797]

18. Inoki K, Li Y, Zhu T, Wu J, Guan KL. TSC2 is phosphorylated and inhibited by Akt and suppresses mTOR signalling. Nat Cell Biol. 2002; 4:648-657. [PubMed: 12172553] 
19. Chi H. Regulation and function of mTOR signalling in T cell fate decisions. Nat Rev Immunol. 2012; 12:325-338. [PubMed: 22517423]

20. Wang Y, et al. Tuberous sclerosis 1 (Tsc1)-dependent metabolic checkpoint controls development of dendritic cells. Proc Natl Acad Sci U S A. 2013; 110:E4894-4903. [PubMed: 24282297]

21. Weichhart T, Hengstschlager M, Linke M. Regulation of innate immune cell function by mTOR. Nat Rev Immunol. 2015; 15:599-614. [PubMed: 26403194]

22. Byles V, et al. The TSC-mTOR pathway regulates macrophage polarization. Nat Commun. 2013; 4:2834. [PubMed: 24280772]

23. Pan H, O'Brien TF, Zhang P, Zhong XP. The role of tuberous sclerosis complex 1 in regulating innate immunity. J Immunol. 2012; 188:3658-3666. [PubMed: 22412198]

24. Fang C, et al. Tsc1 is a Critical Regulator of Macrophage Survival and Function. Cell Physiol Biochem. 2015; 36:1406-1418. [PubMed: 26159807]

25. Hernandez O, Way S, McKenna J 3rd, Gambello MJ. Generation of a conditional disruption of the Tsc2 gene. Genesis. 2007; 45:101-106. [PubMed: 17245776]

26. Prokop S, Heppner FL, Goebel HH, Stenzel W. M2 polarized macrophages and giant cells contribute to myofibrosis in neuromuscular sarcoidosis. Am J Pathol. 2011; 178:1279-1286. [PubMed: 21356378]

27. Misharin AV, Morales-Nebreda L, Mutlu GM, Budinger GR, Perlman H. Flow cytometric analysis of macrophages and dendritic cell subsets in the mouse lung. Am J Respir Cell Mol Biol. 2013; 49:503-510. [PubMed: 23672262]

28. Wong DJ, et al. Module map of stem cell genes guides creation of epithelial cancer stem cells. Cell Stem Cell. 2008; 2:333-344. [PubMed: 18397753]

29. Bertoli C, Skotheim JM, de Bruin RA. Control of cell cycle transcription during G1 and S phases. Nat Rev Mol Cell Biol. 2013; 14:518-528. [PubMed: 23877564]

30. Hochegger H, Takeda S, Hunt T. Cyclin-dependent kinases and cell-cycle transitions: does one fit all? Nat Rev Mol Cell Biol. 2008; 9:910-916. [PubMed: 18813291]

31. Sherr CJ, Beach D, Shapiro GI. Targeting CDK4 and CDK6: From Discovery to Therapy. Cancer Discov. 2015

32. Howell JJ, Ricoult SJ, Ben-Sahra I, Manning BD. A growing role for mTOR in promoting anabolic metabolism. Biochem Soc Trans. 2013; 41:906-912. [PubMed: 23863154]

33. Lebwohl D, et al. Development of everolimus, a novel oral mTOR inhibitor, across a spectrum of diseases. Ann N Y Acad Sci. 2013; 1291:14-32. [PubMed: 23659703]

34. Pforte A, et al. Proliferating alveolar macrophages in BAL and lung function changes in interstitial lung disease. Eur Respir J. 1993; 6:951-955. [PubMed: 8370443]

35. Petzmann S, et al. Enhanced proliferation and decreased apoptosis in lung lavage cells of sarcoidosis patients. Sarcoidosis Vasc Diffuse Lung Dis. 2006; 23:190-200. [PubMed: 18038918]

36. Lan HY, Nikolic-Paterson DJ, Mu W, Atkins RC. Local macrophage proliferation in multinucleated giant cell and granuloma formation in experimental Goodpasture's syndrome. Am J Pathol. 1995; 147:1214-1220. [PubMed: 7485385]

37. Huaux F, et al. IL-1alpha induces CD11b(low) alveolar macrophage proliferation and maturation during granuloma formation. J Pathol. 2015; 235:698-709. [PubMed: 25421226]

38. Sieweke MH, Allen JE. Beyond stem cells: self-renewal of differentiated macrophages. Science. 2013; 342:1242974. [PubMed: 24264994]

39. Robbins CS, et al. Local proliferation dominates lesional macrophage accumulation in atherosclerosis. Nat Med. 2013; 19:1166-1172. [PubMed: 23933982]

40. Haase J, et al. Local proliferation of macrophages in adipose tissue during obesity-induced inflammation. Diabetologia. 2014; 57:562-571. [PubMed: 24343232]

41. Fingar DC, Blenis J. Target of rapamycin (TOR): an integrator of nutrient and growth factor signals and coordinator of cell growth and cell cycle progression. Oncogene. 2004; 23:3151-3171. [PubMed: 15094765]

42. Song J, Salek-Ardakani S, So T, Croft M. The kinases aurora B and mTOR regulate the G1-S cell cycle progression of T lymphocytes. Nat Immunol. 2007; 8:64-73. [PubMed: 17128276] 
43. Miliani de Marval PL, et al. Transgenic expression of cyclin-dependent kinase 4 results in epidermal hyperplasia, hypertrophy, and severe dermal fibrosis. Am J Pathol. 2001; 159:369-379. [PubMed: 11438484]

44. Pearce EL, Pearce EJ. Metabolic pathways in immune cell activation and quiescence. Immunity. 2013; 38:633-643. [PubMed: 23601682]

45. Rodriguez-Prados JC, et al. Substrate fate in activated macrophages: a comparison between innate, classic, and alternative activation. J Immunol. 2010; 185:605-614. [PubMed: 20498354]

46. Hofmann S, et al. Genome-wide association analysis reveals 12q13.3-q14.1 as new risk locus for sarcoidosis. Eur Respir J. 2013; 41:888-900. [PubMed: 22936702]

47. Teirstein AS, et al. Results of 188 whole-body fluorodeoxyglucose positron emission tomography scans in 137 patients with sarcoidosis. Chest. 2007; 132:1949-1953. [PubMed: 17925421]

48. Judson MA, Baughman RP. Worsening of pulmonary sarcoidosis. Curr Opin Pulm Med. 2014; 20:508-516. [PubMed: 25022316]

49. Manzia TM, et al. Successful treatment of systemic de novo sarcoidosis with cyclosporine discontinuation and provision of rapamune after liver transplantation. Transpl Int. 2011; 24:e6970. [PubMed: 21504488]

\section{Supplemental References}

50. Hernandez O, Way S, McKenna J 3rd, Gambello MJ. Generation of a conditional disruption of the Tsc2 gene. Genesis. 2007; 45:101-106. [PubMed: 17245776]

51. Rosner M, et al. Efficient siRNA-mediated prolonged gene silencing in human amniotic fluid stem cells. Nat Protoc. 2010; 5:1081-1095. [PubMed: 20539284] 

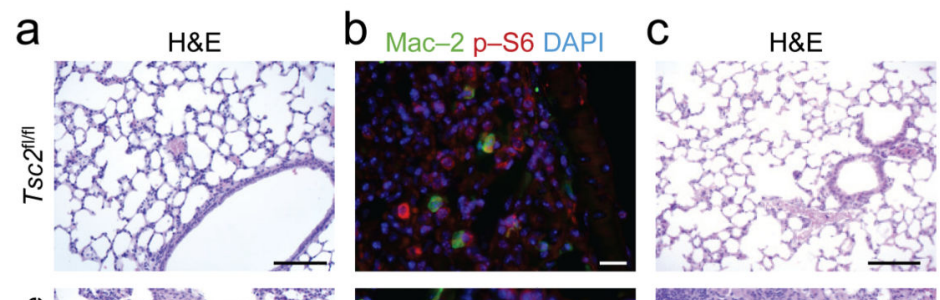

d
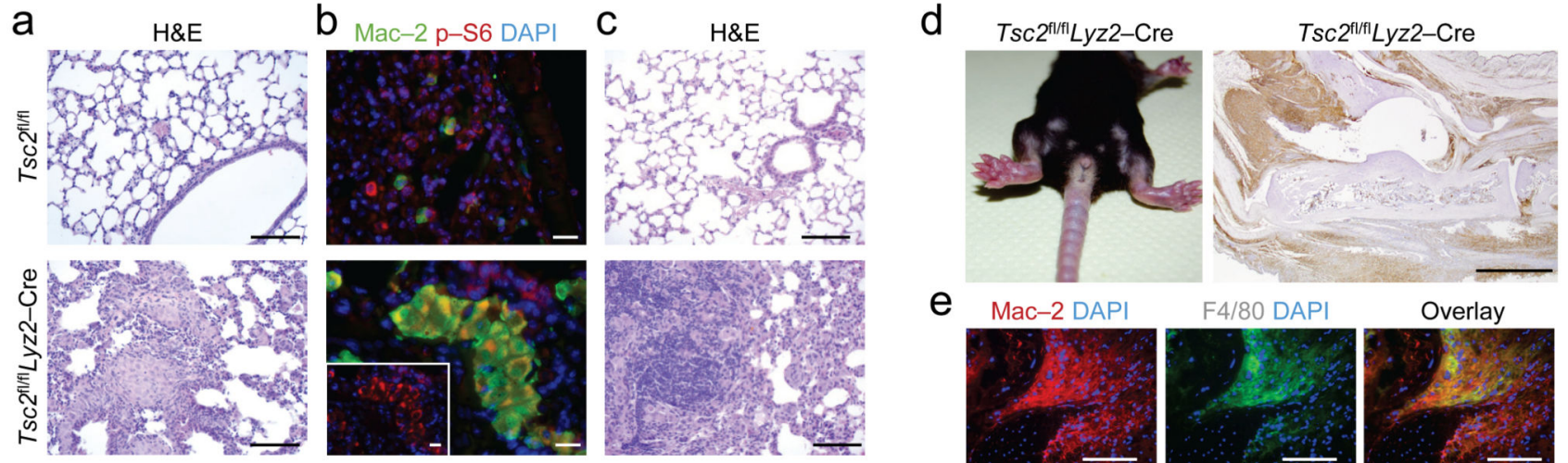

\section{e}
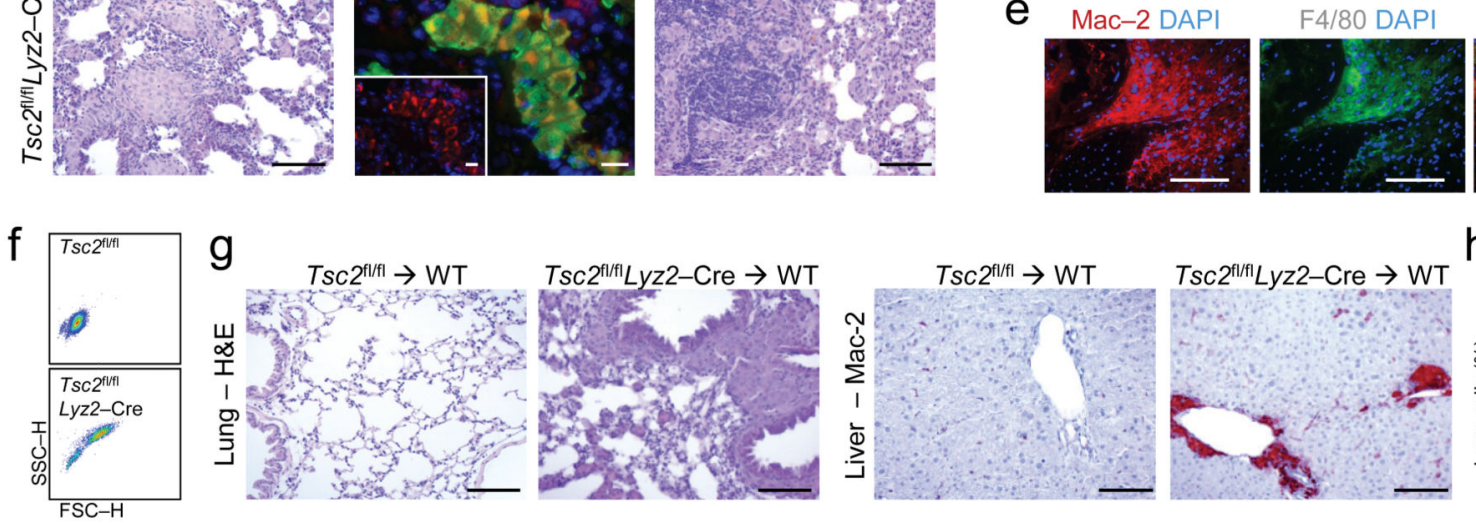

$h=T s c c^{\text {2ut }}$ - Tsc2 2"ritLyz2-Cre

i
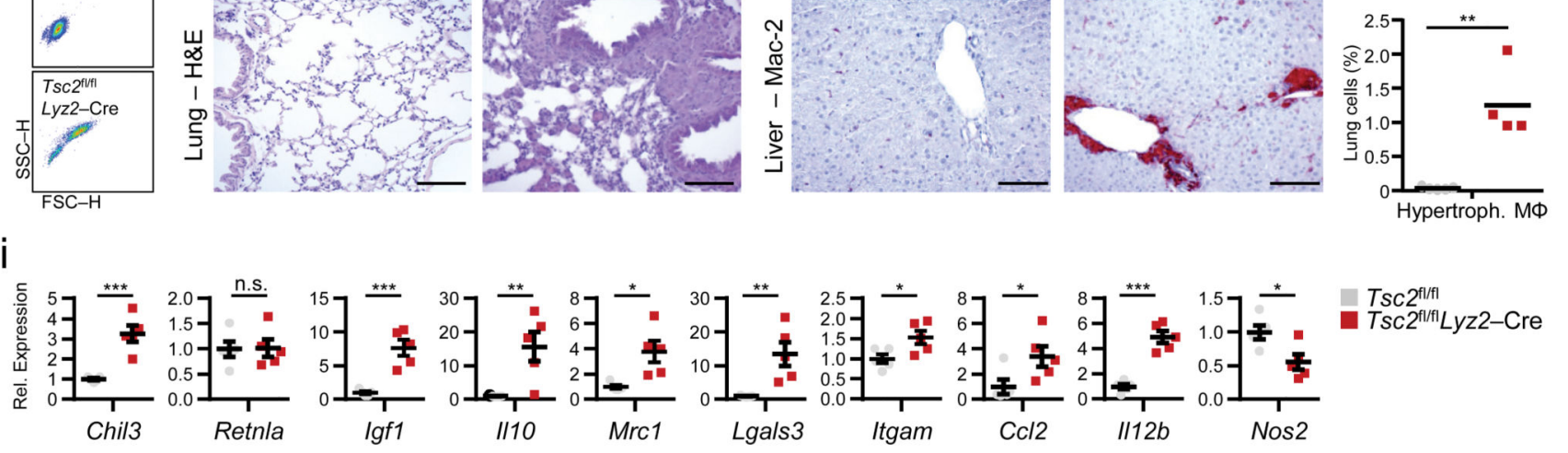

Figure 1. Hypertrophic M2-like macrophages spontaneously form granulomas in $T_{s c 2} 2^{\mathrm{fl} / \mathrm{fl}} \mathrm{Lyz2}$ Cre mice.

(a,c) Lung sections evaluated by H\&E staining of $T_{s c} 2^{\mathrm{fl} / \mathrm{fl}}$ and $T_{s c} 2^{\mathrm{fl} / \mathrm{fl}} L y z 2-\mathrm{Cre}$ mice at the age of (a) 3 and (c) 6 months. (b) Immunofluorescence for Mac-2 and p-S6 in lung sections of 3 month-old mice. Boxed region shows p-S6 staining only. (d) Image of a $T s c 2^{\text {fl/fl } L y z 2-}$ Cre mouse at the age of 6 months (left) and immunohistochemistry of Mac-2 of a paw (right). (e) Immunofluorescence for Mac-2 and F4/80 in paw sections of 6 month-old $T s c 2^{1 / / 1 / 1} L y z 2-C r e$ mice. (f) Flow cytometric scatterplot of the G1 population of $T s c 2^{1 / / f 1}$ and $T s c 2^{\mathrm{fl} / \mathrm{fl}} L y z 2-C r e$ peritoneal macrophages. (g) $T s c 2^{\mathrm{fl} / \mathrm{fl}}$ and $T s c 2^{\mathrm{fl} / \mathrm{fl}} L y z 2$-Cre bone marrow was injected into irradiated wild-type recipient mice and evaluated after 3 months by histology. Left panel: H\&E staining of lung sections. Right panel: IHC of Mac-2 in liver. (h) Flow cytometry analysis of hypertrophic macrophages in the lungs of $T s c 2^{\mathrm{fl} / \mathrm{fl}}(\mathrm{n}=5)$ and $T s c 2^{11 / f 1}$ Lyz2-Cre $(n=4)$ mice. Shown are means. (i) Expression of the indicated mRNAs in the lungs of 6 month-old mice. Shown are means $\pm \operatorname{SE}(n=5)$. ${ }^{*}<<0.05$, **p $<0.01$, ***p $<$ 0.001 ; n.s., not significant (Student's $t$ test). Representative histological images or plots are from one mouse; each analysis was performed on three $(\mathbf{b}, \mathbf{d}, \mathbf{e}, \mathbf{g})$ or five $(\mathbf{a}, \mathbf{c}, \mathbf{f})$ mice per genotype. Numerical data are representative of two independent (h) or cumulative of two independent (i) experiments. Scale bars, $100 \mu \mathrm{m}(\mathbf{a}, \mathbf{c}, \mathbf{e}, \mathbf{g}), 10 \mu \mathrm{m}(\mathbf{b}), 1 \mathrm{~mm}$ (d). 
a

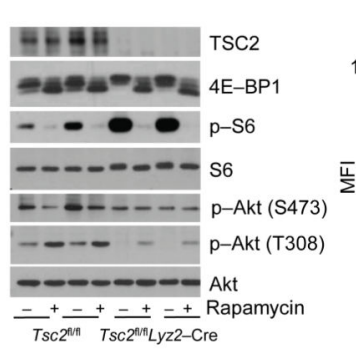

b

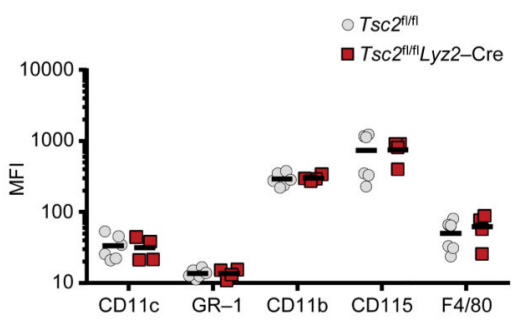

C

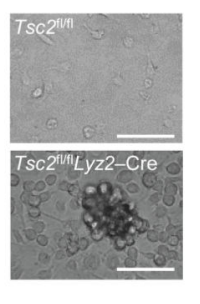

d

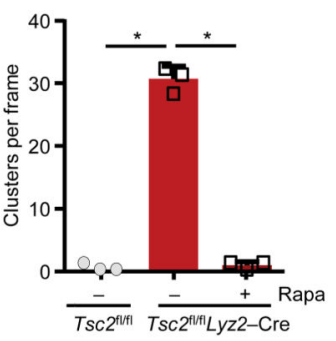

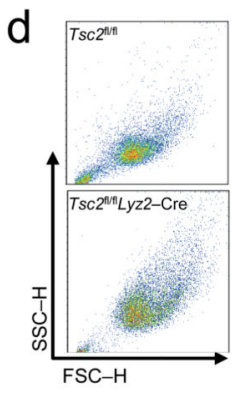
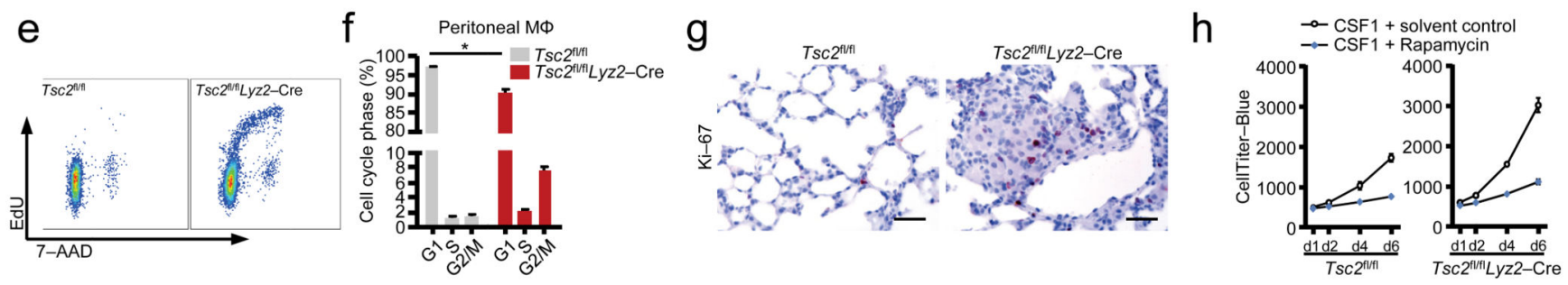

Figure 2. TSC2 inhibits granulomatous aggregation, hypertrophy and macrophage proliferation in vitro.

(a) Immunoblots of CSF1-deprived $T s c 2^{\mathrm{fl} / \mathrm{fl} 1}$ and $T s c 2^{\mathrm{fl} / / \mathrm{f} 1} L y z 2$-Cre bone marrow-derived macrophages (BMDM), treated for 18 hours with $100 \mathrm{nM}$ rapamycin or solvent control, hybridized with the indicated antibodies. (b) Surface expression of the indicated proteins on $T s c 2^{\mathrm{l} l / f l}(\mathrm{n}=6)$ and $T s c 2^{\mathrm{fl} / \mathrm{fl}} L y z 2$-Cre $(\mathrm{n}=4)$ BMDM after differentiation. (c) Left: Images of IL-4 stimulated (10 ng/ml) $T s c 2^{\mathrm{fl} / \mathrm{fl}}$ and $T s c 2^{\mathrm{fl} / \mathrm{fl}} L y z 2$-Cre BMDM treated with solvent or $100 \mathrm{nM}$ rapamycin for four days. Right: Quantification of cluster formation. Clusters in three pictures per condition ( $4 x$ magnification) were counted $(n=3)$. (d) Flow cytometry scatterplot of CSF1-deprived BMDM on day 7. (e,f) Cell cycle analysis of (e) BMDM by 7AAD and EdU staining and of (f) peritoneal macrophages by PI staining from $T s c 2^{\mathrm{fl} / \mathrm{fl}}$ and $T s c 2^{\mathrm{fl} / \mathrm{fl}} \operatorname{Lyz2}$-Cre mice $(\mathrm{n}=5)$. (g) IHC of Ki-67 in lung sections of 3 month-old $T s c 2^{2 \mathrm{l} / / \mathrm{fl}}$ and $T s 2^{21 / 1 / 1} L y z 2$-Cre mice. (h) Proliferation analysis of BMDM stimulated with $40 \mathrm{ng} / \mathrm{ml} \mathrm{CSF1}$ and treated with solvent or $100 \mathrm{nM}$ rapamycin $(\mathrm{n}=4)$. Shown are means $\pm \mathrm{SE}(\mathbf{b}, \mathbf{f}, \mathbf{h})$ or SD (c). ${ }^{*} \mathrm{p}<0.001$ (Student's $t$ test) $(\mathbf{c}, \mathbf{f})$. Data are representative of three $(\mathbf{a}, \mathbf{d}, \mathbf{g}, \mathbf{c})$ or two (e) or cumulative from two independent experiments (b,f,h) Scale bar, $100 \mu \mathrm{m}(\mathbf{c}), 50 \mu \mathrm{m}(\mathrm{g})$. 
a

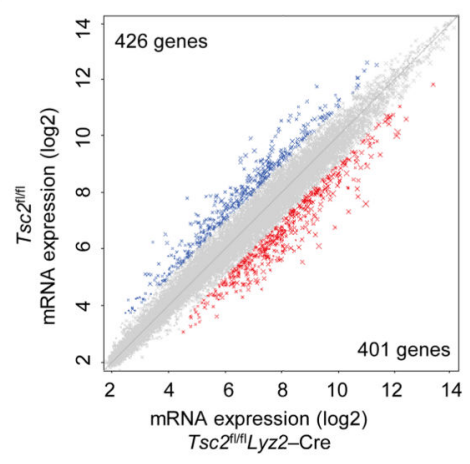

d

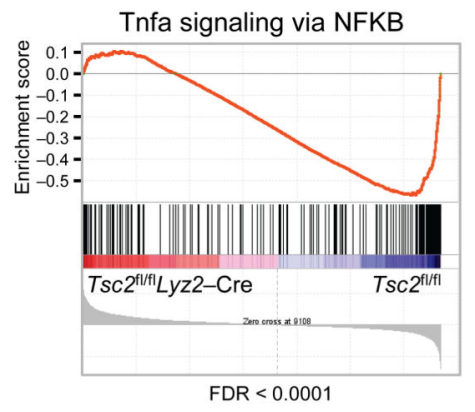

b

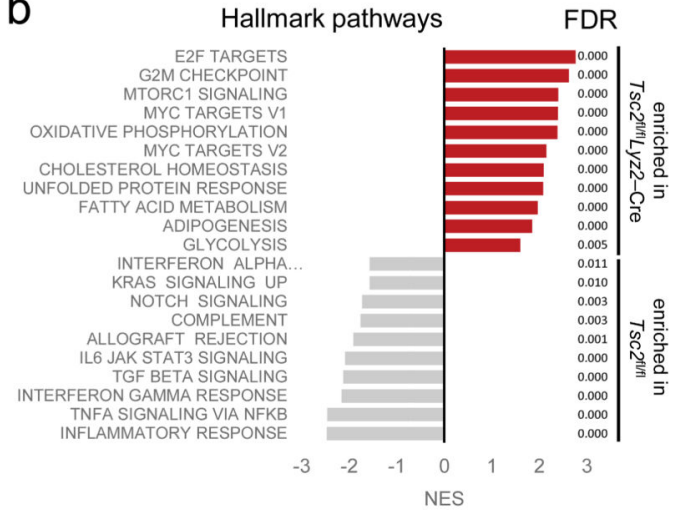

C

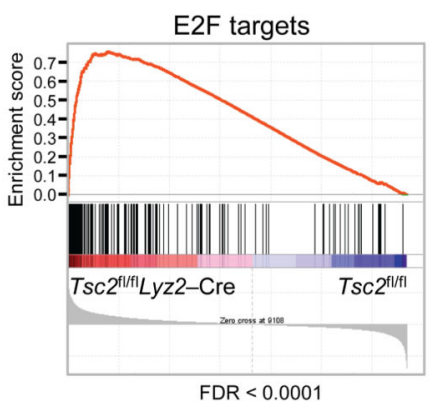

e

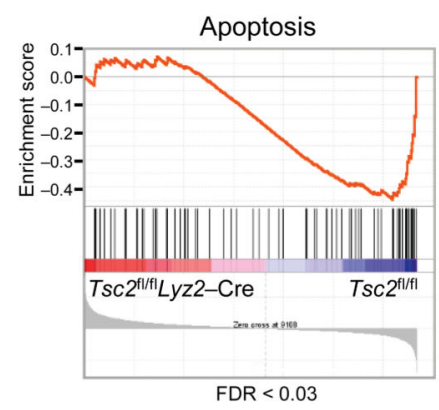

$f$

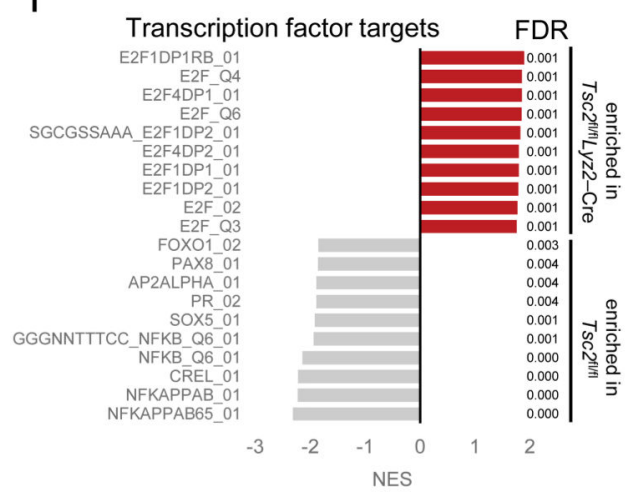

Figure 3. TSC2 globally regulates macrophage proliferation, apoptosis and inflammation.

(a) Scatterplot of global gene-expression profiles of CSF1-deprived $T_{S c} 2^{\mathrm{fl} / \mathrm{fl}}$ and $T_{s c} 2^{\mathrm{fl} / \mathrm{fl}}$ Lyz2-Cre BMDM. Blue dots, 426 genes significantly higher expressed in Tsc $2^{\mathrm{fl} / \mathrm{fl}}$ BMDM; red dots, 401 genes significantly higher expressed in $T_{s c} 2^{\mathrm{fl} / \mathrm{fl}}$ Lyz2-Cre BMDM. (b) Gene-set enrichment analysis (GSEA) of hallmark gene sets (H.all) from the Molecular Signatures Database of the Broad Institute, showing the most significantly enriched gene sets in $T s c 2^{\mathrm{fl} / \mathrm{fl}}$ and $T s c 2^{\mathrm{fl} / \mathrm{fl}}$ Lyz2-Cre BMDM and their normalized enrichment scores (NES) as well as their false discovery rates (FDR). (c,d) GSEA plot of the E2F targets (c) and Tnfa signaling via NFKB (d) gene signature in $T_{s c} 2^{\mathrm{fl} / \mathrm{fl}} L y z 2-C r e$ BMDM relative to $T_{s c} 2^{\mathrm{fl} / \mathrm{fl}}$ BMDM from the analysis in (b) with members of the gene set presented in the ranked list of genes ('bar code' below) and the signal-to-noise ranking metric (bar at bottom). (e) GSEA of the 'KEGG_apoptosis' gene signature in $T s c 2^{\mathrm{fl} / \mathrm{fl}} L y z 2-\mathrm{Cre}$ BMDM relative to $T s c 2^{\mathrm{fl} / \mathrm{fl}}$ BMDM. (f) The most significantly enriched transcription factor target gene sets (C3.TFT) from the Molecular Signatures Database in unstimulated Tsc $2^{\mathrm{fl} / \mathrm{fl}}$ and $T s c 2^{\mathrm{fl} / \mathrm{fl}} L y z 2-\mathrm{Cre}$ BMDM derived by GSEA. NES, normalized enrichment score; FDR, false discovery rate. Data are from one experiment with four biological replicates per genotype obtained from two independent experiments. 
a

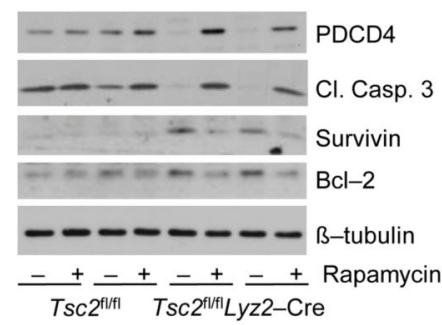

b

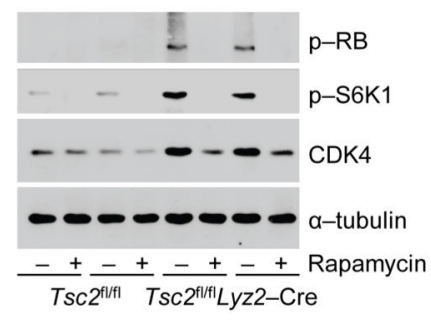

C

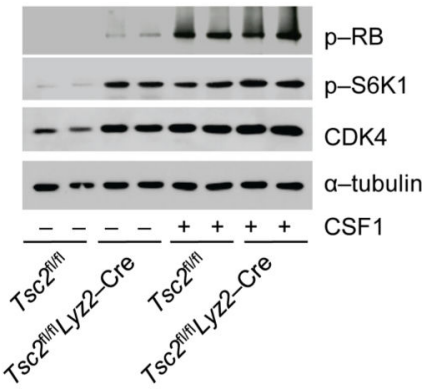

d

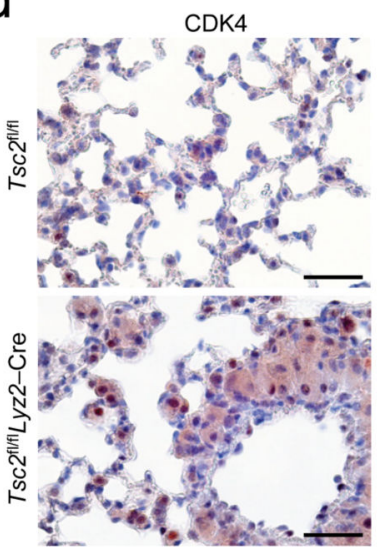

f

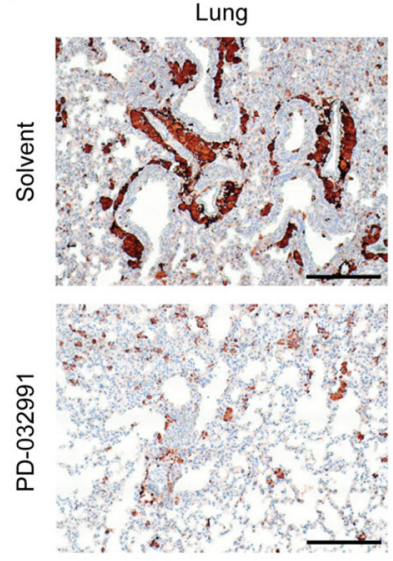

e
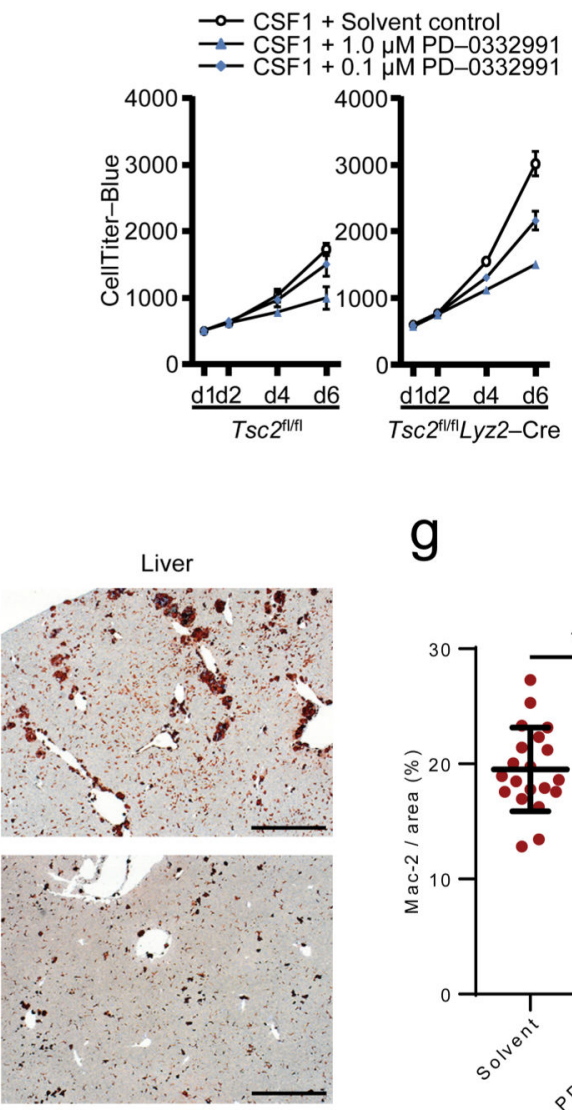

g

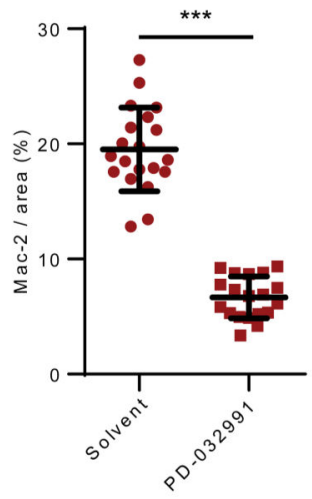

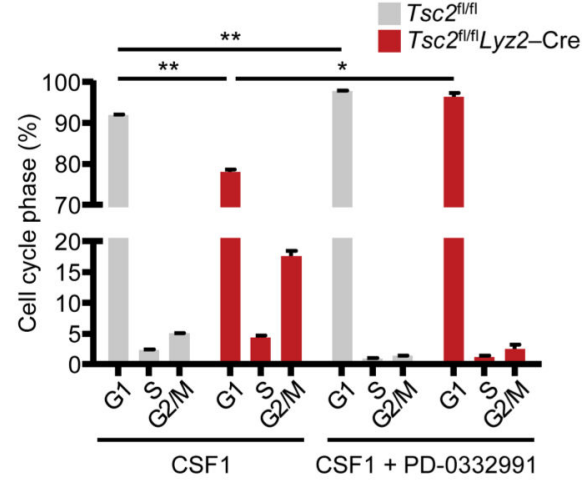

h

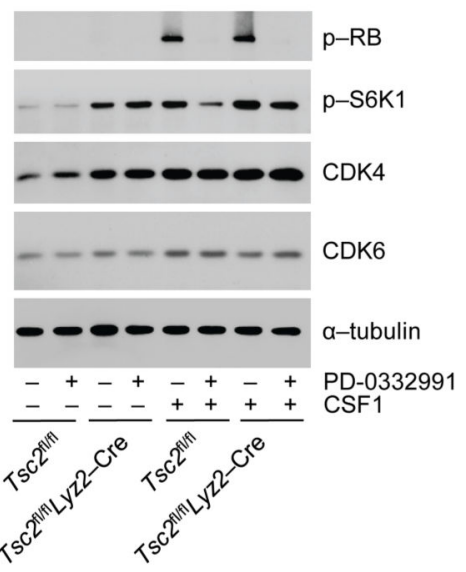

Figure 4. CSF1 induces CDK4 expression via TSC2/mTORC1 in macrophages.

(a,b,c) BMDM were treated with $100 \mathrm{nM}$ rapamycin, solvent control and $10 \mathrm{ng} / \mathrm{ml} \mathrm{CSF1}$ as indicated for $18 \mathrm{~h}$. Whole cell lysates were analyzed by immunoblotting with the indicated antibodies. (d) IHC of CDK4 in lung sections of 3 month-old mice. (e) Left, analysis of CSF1-induced $(40 \mathrm{ng} / \mathrm{ml})$ proliferation of BMDM treated with solvent or the indicated amounts of the CKD4 inhibitor PD-0332991 ( $n=4)$. Right, cell cycle analysis of CSF1stimulated BMDM treated with solvent or $1 \mu \mathrm{M}$ PD-0332991 for $18 \mathrm{~h}(\mathrm{n}=3)$. (f,g) $T s c 2^{1 / / f 1} L y z 2-C r e$ bone marrow was injected into irradiated wild-type recipient mice. After 
ten days mice were treated daily with PD-0332991 $(n=10)$ or solvent control $(n=10)$ daily for two month. (f) Lung (left panel) and liver (right panel) sections were evaluated by IHC of Mac-2. (g) Area of Mac-2 positive cells compared to total lung area of the treated mice. Two random images per animal were evaluated. (h) BMDM were treated with $1 \mu \mathrm{M}$ PD-0332991 or solvent control and stimulated with $10 \mathrm{ng} / \mathrm{ml} \mathrm{CSF} 1$ as indicated for $18 \mathrm{~h}$. Whole cell lysates were analyzed by immunoblotting with the indicated antibodies. Shown are means \pm $\mathrm{SE}(\mathbf{e})$ or SD $(\mathbf{g}) . * \mathrm{p}<0.01,{ }^{*} \mathrm{p}<0.001$ (Student's $t$ test). Data are representative of three $(\mathbf{a}, \mathbf{b}, \mathbf{c}, \mathbf{d})$, one (f,g) or two (h) independent, or cumulative of two (e) experiments. Scale bar, $40 \mu \mathrm{m}(\mathbf{d}), 100 \mu \mathrm{m}(\mathbf{f})$. 

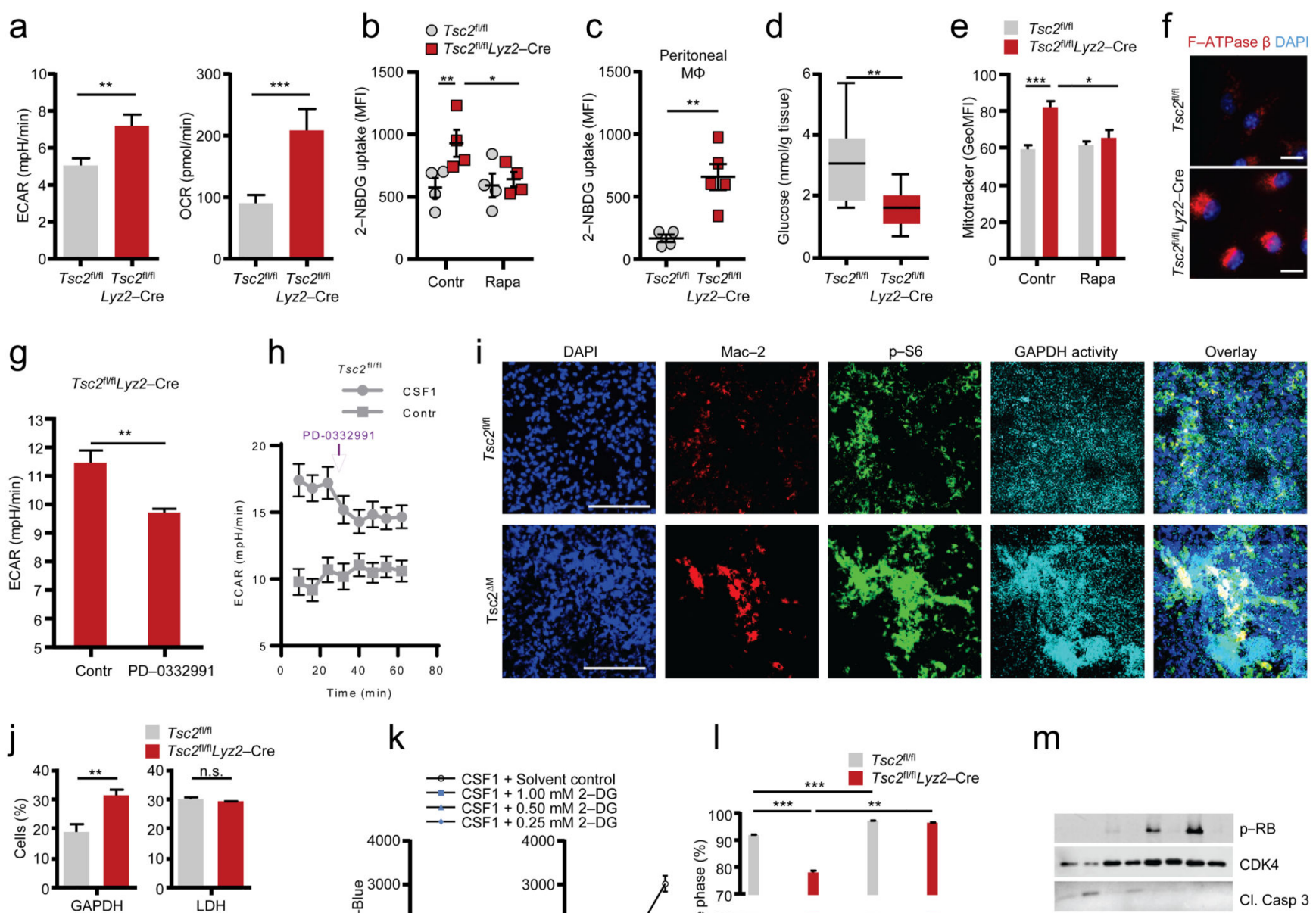

$T s c 2^{\mathrm{flf} / \mathrm{fl}}$
$T s c 2^{\mathrm{fl} / \mathrm{f}} L y z 2-\mathrm{Cre}$
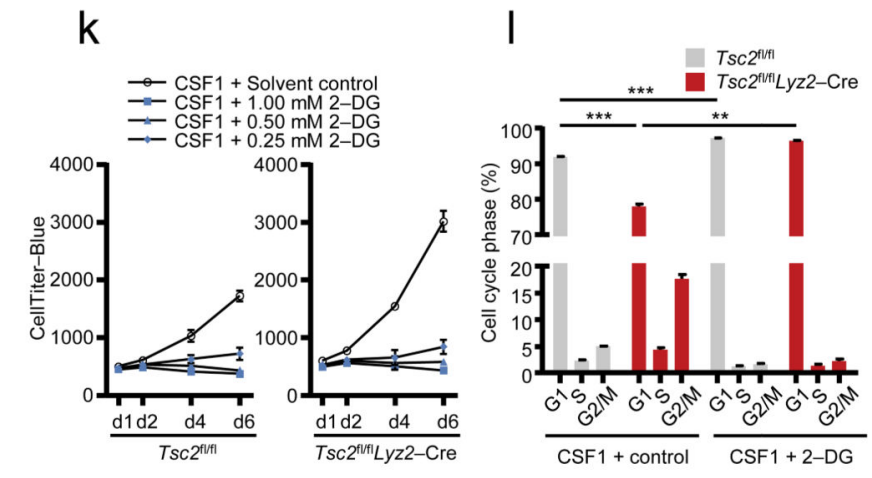

$m$
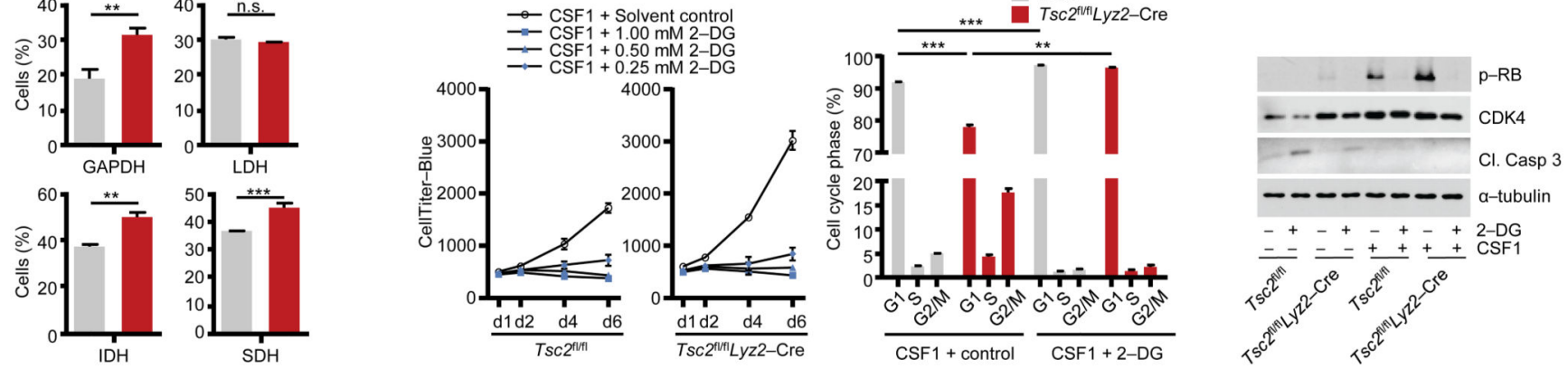

Figure 5. TSC2 regulates the cellular metabolism in macrophages via CDK4 to promote proliferation.

(a) Basal extracellular acidification rate (ECAR) and oxygen consumption rate (OCR) of BMDM reported in units of $\mathrm{mpH} / \mathrm{min}$ and $\mathrm{pmol} / \mathrm{min}$, respectively $(\mathrm{n}=8)$. (b) Uptake of 2NBDG in BMDM treated with solvent control or $100 \mathrm{nM}$ rapamycin for $18 \mathrm{~h}$; analyzed by flow cytometry $(\mathrm{n}=4)$. (c) Uptake of 2-NBDG in peritoneal macrophages from $\mathrm{Tsc}^{\mathrm{fl} / \mathrm{fl}}(\mathrm{n}=4)$ and $T s c 2^{[1 / f 1} L y z 2$-Cre ( $\left.\mathrm{n}=5\right)$ mice. (d) Total amount of glucose in lung tissue of mice $(\mathrm{n}=10)$. (e) Mitotracker Green staining analyzed by flow cytometry of BMDM treated with solvent control or $100 \mathrm{nM}$ rapamycin for $18 \mathrm{~h}(\mathrm{n}=4)$. (f) Immunofluorescence for F-ATPase $\beta$ of BMDM deprived of CSF1. (g) ECAR of $T s c 2^{\text {fl/fl }}$ Lyz2-Cre BMDM that were treated for 30 min with solvent or $1 \mu \mathrm{M}$ PD-0332991 $(\mathrm{n}=8)$. (h) ECAR of $T s c 2^{\mathrm{fl} / \mathrm{fl}}$ that were treated overnight with solvent or $10 \mathrm{ng} / \mathrm{ml} \mathrm{CSF} 1$ and at the indicated time point with $1 \mu \mathrm{M}$ PD-0332991 (n=8). (i) Images of glyceraldehyde 3-phosphate dehydrogenase (GAPDH) activity on frozen lung sections of mice in situ. Sections were additionally stained for Mac-2, p-S6, and DAPI. (j) Fraction (in \%) of p-S6-positive Mac-2 macrophages in lungs of 
mice with high activities of GAPDH, lactate dehydrogenase (LDH), isocitrate dehydrogenase (IDH) and succinate dehydrogenase (SDH) (n=3-4). (k) Analysis of CSF1induced $(40 \mathrm{ng} / \mathrm{ml})$ proliferation of BMDM treated with solvent or the indicated amounts of 2-DG $(n=4)$. (l) Cell cycle analysis of CSF1-stimulated BMDM treated with solvent or 1 $\mathrm{mM}$ 2-DG for $18 \mathrm{~h}(\mathrm{n}=3)$. (m) BMDM were treated with $1 \mathrm{mM}$ 2-DG or solvent control and then stimulated with $10 \mathrm{ng} / \mathrm{ml} \mathrm{CSF1}$ for $18 \mathrm{~h}$. Whole cell lysates were analyzed by immunoblotting with the indicated antibodies. Shown are means \pm SD $(\mathbf{a}, \mathbf{g}, \mathbf{h}, \mathbf{j})$ or SE (b,c,e,k,l) or boxplots with means, $25^{\text {th }}$ to $75^{\text {th }}$ percentile and minimum to maximum bars (d). ${ }^{*} \mathrm{p}<0.05, * * \mathrm{p}<0.001,{ }^{* * *} \mathrm{p}<0.001$ (Student's $t$ test). Data are representative of one (d) or two $(\mathbf{a}, \mathbf{f}-\mathbf{j}, \mathbf{m})$ independent experiments or cumulative of 2 experiments (b,c,e,k,l). Scale bar, $10 \mu \mathrm{m}(\mathbf{f}), 100 \mu \mathrm{m}(\mathbf{i})$. 


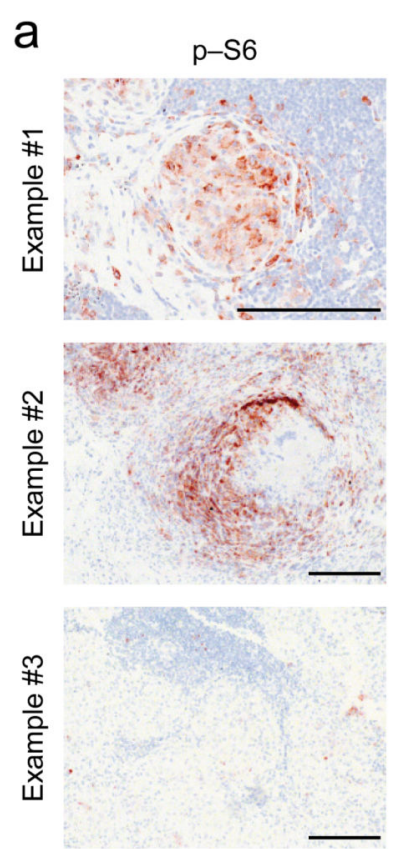

b
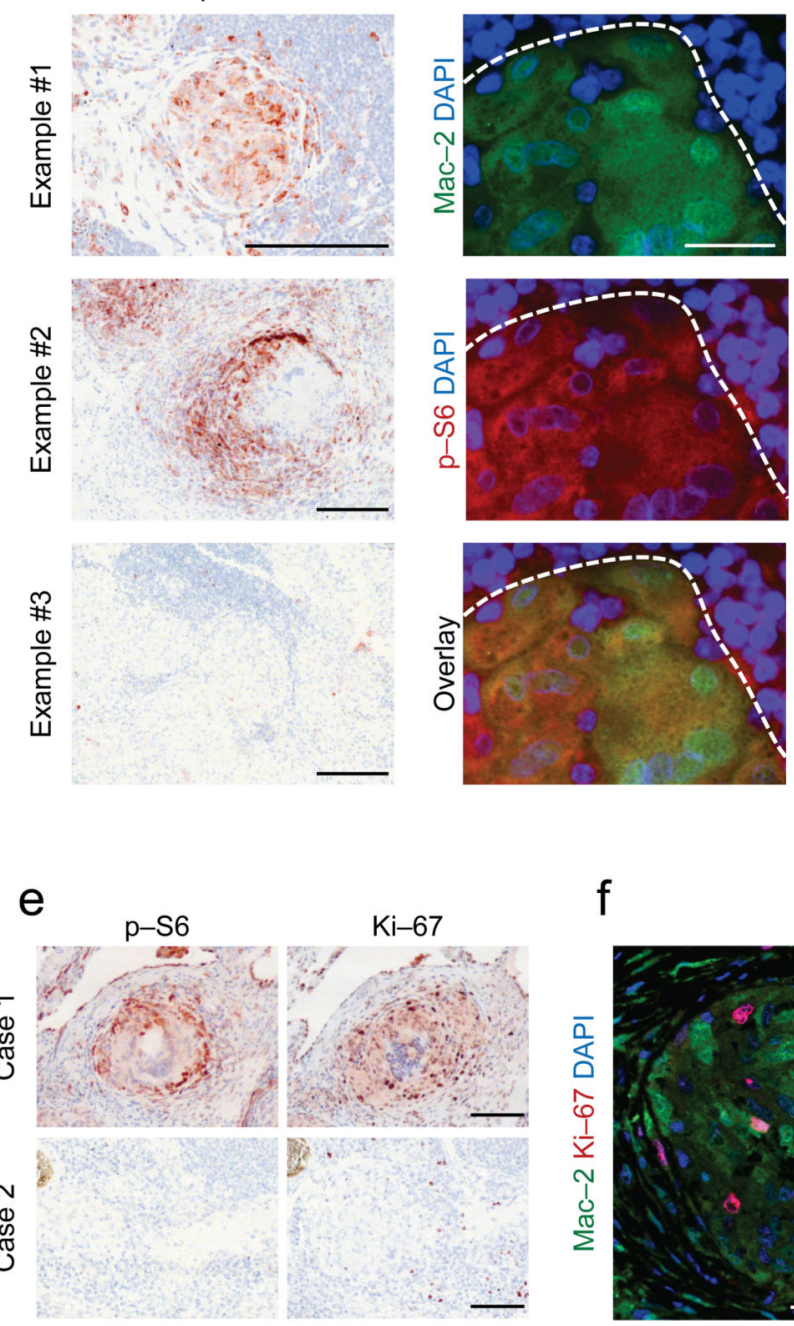
proliferation.
C
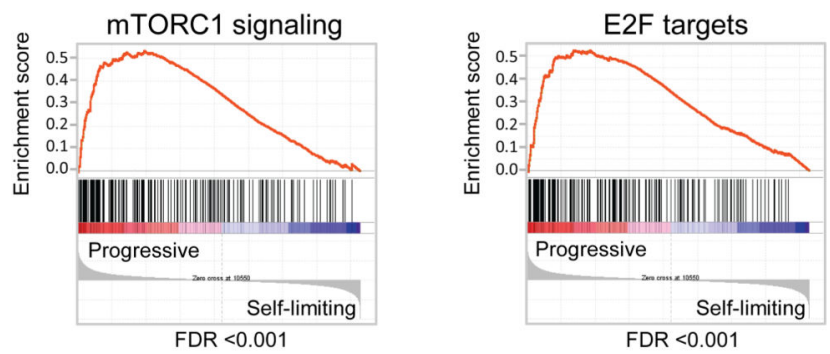

d

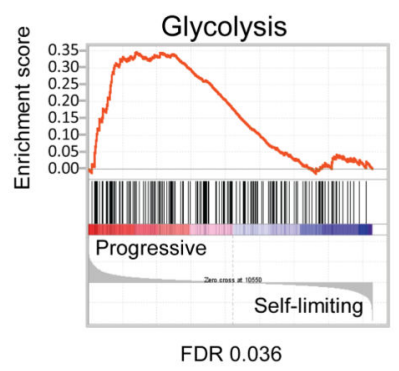

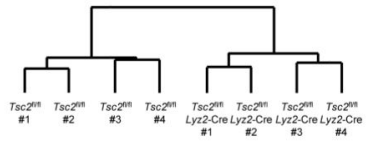

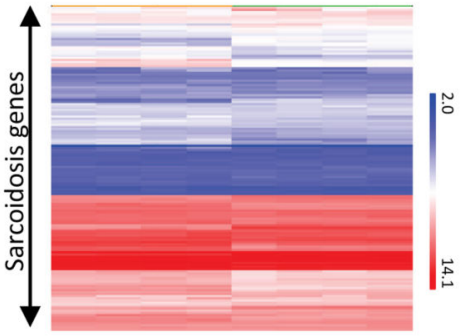

Figure 6. Disease progression in human sarcoidosis is associated with mTORC1 signaling and

(a) Sections of three sarcoidosis patients stained with p-S6 by IHC. (b) Immunofluorescence for Mac-2 and p-S6 in a sarcoidosis granuloma. (c) GSEA analysis of the 'mTORC1 signaling', 'E2F targets', and 'Glycolysis' gene signatures in progressive relative to selflimiting sarcoidosis from the data of Lockstone et al9. (d) An unsupervised cluster analysis of the microarray data of $T s c 2^{[1 / f 1}$ and $T s c 2^{f l / f l} L y z 2$-Cre BMDM (Fig. 3a) performed with the genes that were differentially expressed in progressive relative to self-limiting sarcoidosis patients. (e) IHC for p-S6 and Ki-67 of granulomas from sarcoidosis patients. (f) Immunofluorescence for Mac-2 and Ki-67 in a sarcoidosis granuloma. (g) Relationship between p-S6 and Ki-67 expression in granulomas of 27 human sarcoidosis biopsies. Ki-67 high, > $5 \%$ Ki-67 positive cells in the granuloma; Ki-67 low, $<5 \%$ Ki-67 positive cells in the granulomas. The relationship was investigated using fisher's exact test. Data are representative of 27 human sarcoidosis patients (a,b,e,f) or from 8 self-limiting and 7 progressive sarcoidosis patients (c,d). Scale bar, $200 \mu \mathrm{m}$ (a), $20 \mu \mathrm{m}$ (b), $100 \mu \mathrm{m}(\mathbf{e , f})$. 
a
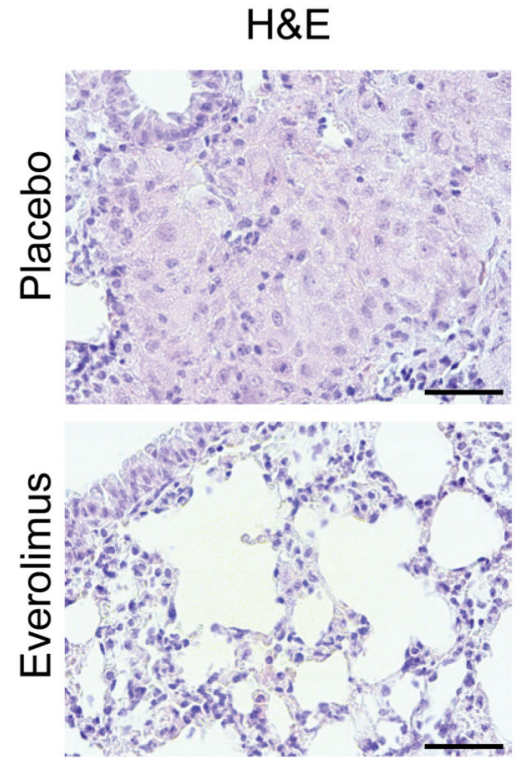

d
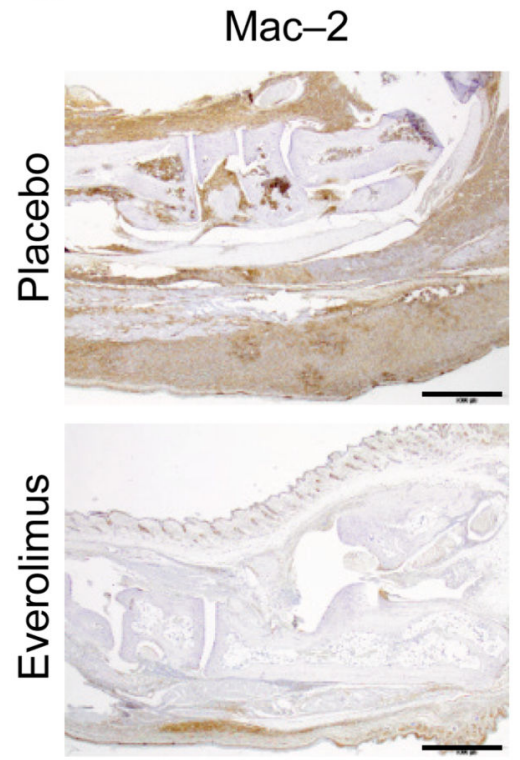

b
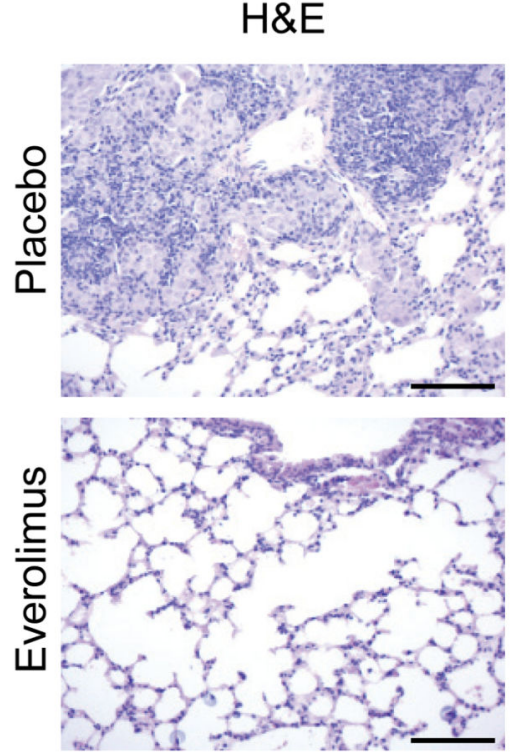

e

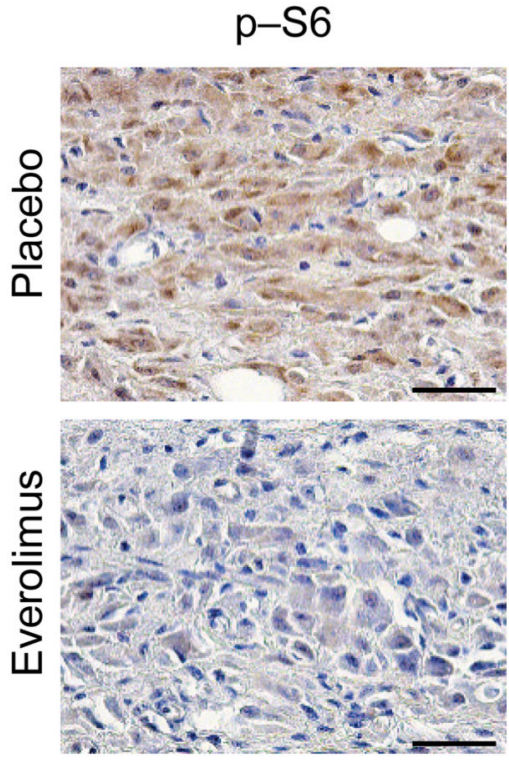

C
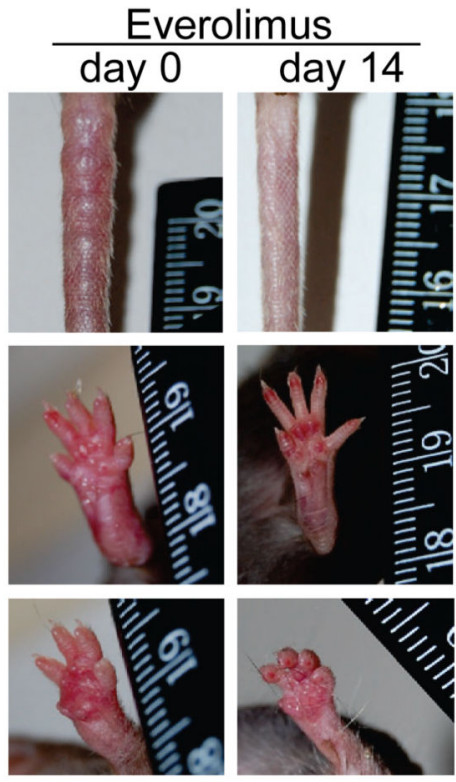

\section{Cleaved caspase 3}

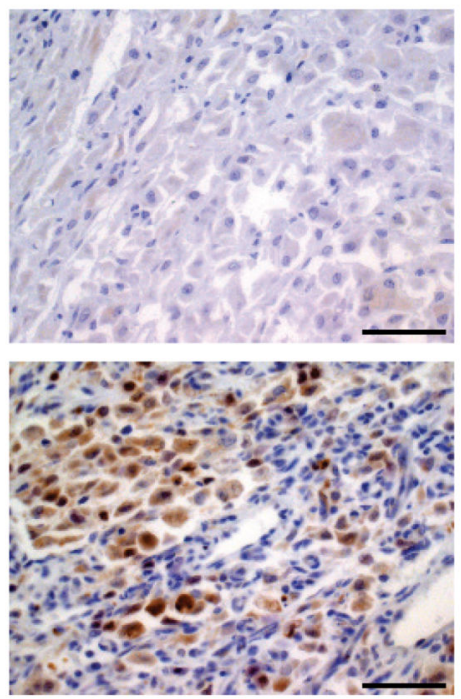

Figure 7. Inhibition of mTORC1 restores homeostasis in $T s c 2^{\mathrm{f} / \mathrm{fl}} L y z 2$-Cre mice.

(a) 2 month-old and (b) 6 month-old $T s c 2^{\text {fl/f1 }}$ Lyz2-Cre mice were treated with placebo or everolimus for three weeks and lung sections were evaluated by H\&E staining. (c) Paw and tail images of a 6 month-old $T s c 2^{21 / 1 / 1} L y z 2$-Cre mouse at day 0 and 14 of everolimus treatment. (d) IHC for Mac-2 of paw sections of 6 month-old $T s c 2^{\mathrm{fl} / \mathrm{fl}} L y z 2$-Cre mice treated with placebo or everolimus. (e) IHC for p-S6 and cleaved caspase 3 of paw sections of 6 month-old $T s c 2^{\mathrm{fl} / \mathrm{fl}} \mathrm{Lyz2}$-Cre mice treated with placebo or everolimus for two days.

Representative histological images are from one mouse; each analysis was performed on 
four $(\mathbf{a}, \mathbf{b}, \mathbf{c})$ or three $(\mathbf{d}, \mathbf{e})$ mice per genotype. Scale bar, $40 \mu \mathrm{m}(\mathbf{a}), 100 \mu \mathrm{m}(\mathbf{b}), 1 \mathrm{~mm}(\mathbf{d}), 50$ $\mu \mathrm{m}(\mathbf{e})$. 